\title{
Consideration of generated beam angles increases the accuracy of ultrasonic displacement measurements
}

This article was published in the following Dove Press journal:

Reports in Medical Imaging

8 March 2012

Number of times this article has been viewed

\author{
Chikayoshi Sumi \\ Yuuki Takanashi \\ Kento Ichimaru \\ Department of Information and \\ Communication Sciences, Faculty \\ of Science and Technology, Sophia \\ University, Tokyo, Japan
}

Correspondence: Chikayoshi Sumi Department of Information and Communication Sciences, Faculty of Science and Technology, Sophia University, 7-I Kioi-cho, Chiyoda-ku, Tokyo 102-8554, Japan

$\mathrm{Tel}+8|3323834| 5$

Fax +8I 33238332

Email c-sumi@sophia.ac.jp
Abstract: The development of practical ultrasonic (US) tissue displacement measurement methods increases the number of available and useful applications of displacement/strain measurements that can be made (eg, various blood flow measurements and measurements of tissue motion in organs such as the heart, liver, and so forth). Previously developed lateral modulation (LM) methods with a multidimensional autocorrelation method (MAM) or multidimensional Doppler method (MDM) and a steering angle method (ASTA) with lateral Doppler method produced accurate displacement vector and lateral displacement measurements, respectively. Such measurements cannot be obtained using only a conventional Doppler technique. Another new method has also been reported, using multiple crossed beams (MCBs) to obtain high-accuracy displacement vector measurements; that is, a displacement vector is synthesized using accurately measured axial displacements with previously developed multidimensional displacement measurement methods, including the one-dimensional autocorrelation method (1D AM) with a multidimensional moving average (MA), together with conventional rotation processing of global echo data or a coordinate system (ie, a global echo rotation referred to as $\mathrm{r}$ method) by the negative value of the steering angles used in beamforming. However, in real-world applications, directivities of transmission and reception apertures, scattering, reflection, and attenuation affect the direction and properties of US beams used for conventional axial displacement measurements employing beamforming methods such as a conventional nonsteered, steered, or secta beam, and they also affect ASTA and MCB methods. In this report, to improve accuracy in the measurements of an arbitrary directional displacement and a displacement vector using any beamforming methods, a spatial resolution in a beam angle (BA) is generated. For instance, for a two-dimensional (2D) Cartesian coordinate system, this is obtained by calculating the arctangent of the ratio of the axial and lateral instantaneous frequencies or the first moments of local spectra. On the basis of the 1D AM with a multidimensional MA, the local displacement in the beam direction is accurately measured by dividing the local instantaneous phase change by the instantaneous frequency calculated in the beam direction, and an arbitrary directional displacement can be measured (axial, lateral, radial, and so forth), which is done with or without a rotation of local echo data. These are respectively referred to as BA and BAr (BA + local rotation) methods or, specifically, as the 1D AMBA (1D AM + BA) and the 1D AMBAr (1D AM + BAr). Also, it is theoretically shown that the 1D AM with MCB but no echo rotation (ie, 1D AMBA with MCB) is equivalent to the most accurate MAM with LM, and that the 1D AMBA with ASTA can also provide a lateral Doppler measurement. Through agar phantom experiments, in addition to steered spherical focusing beams for both transmission and reception, measurement accuracies with all of the new methods are also evaluated for rapid scanning beamforming such as using one or plural, steered or nonsteered plane wave transmissions, and steered or nonsteered spherical focusing beam receptions. For comparisons with the 1D AMBA and the 1D AMBAr, the spectra frequency division method (SFDM) used in the MAM is also used 
instead of the 1D AM. All for the measurements of an axial displacement with axial compression and nonsteering, a lateral displacement with lateral compression and ASTA and a 2D displacement vector with lateral compression and MCBs, BA methods based on the 1D AM and SFDM approaches achieve more accurate measurements with significantly fewer calculations than the corresponding BAr or $r$ methods (ie, real time). BA methods do not yield dead region data in a region of interest and they do not yield any measurement errors because of an approximate interpolation of echo data or of measured motion/deformation data. However, the measurement accuracies of one-directional displacements are significantly lower than those of displacement vectors, because of the practical three-dimensional deformation or motion (eg, about a half standard deviation of the lateral displacement measurement). In terms of required processing, the MAM or the MDM with LM is better than methods with MCB (ie, real time). The proper combination of the SFDM with LM or ASTA will yield more accurate measurements in a trade-off with increasing the number of calculations.

Keywords: beam direction, beam angle (BA), axial displacement, lateral displacement, displacement vector, lateral modulation (LM), a steering angle (ASTA), multiple crossed beams (MCBs), multidimensional or one-dimensional autocorrelation method (MAM or 1DAM), multidirectional moving-average (MA), spectra frequency division method (SFDM)

\section{Introduction}

Various ultrasonic (US) displacement/velocity measurement methods have been extensively developed for measurements of blood flow (eg, the continuous wave Doppler method $[\mathrm{DM}],{ }^{1}$ the pulse wave $\mathrm{DM},{ }^{2}$ the autocorrelation method $[\mathrm{AM}],{ }^{3}$ the cross-correlation method $[\mathrm{CCM}]^{4}$ ), and tissue strain (eg, DM, AM, ${ }^{5}$ and $\mathrm{CCM}^{6}$ ). Methods have also been developed for the analysis of sonar data and for other target motions. For approximately the past 60 years, tissue axial displacement/velocity/strain has been measured using such methods (ie, they are one-dimensional [1D] measurement methods), whereas other developments have permitted measurements of multidimensional displacement/velocity vectors and of strain/strain-rate tensors for blood flow ${ }^{7}$ (in the heart and other organs) and tissue motion (in the liver, and so forth $)^{8}$ (ie, the multidimensional CCM [MCCM]).

Sumi et $\mathrm{al}^{9-19}$ have also developed other displacement measurement methods. For instance, the multidimensional cross-spectrum phase gradient method (MCSPGM), ${ }^{9,10}$ the multidimensional autocorrelation method (MAM), ${ }^{11-13}$ and the multidimensional DM (MDM) ${ }^{11-13}$ were developed for displacement vector measurements - specifically, for simultaneous axial, lateral, and elevation displacement measurements. Such methods will be applied to measurements of blood flow and motion/deformation of the heart, blood vessels, liver, skin, muscle, and so forth under normal spontaneous motion or under artificial motion (static compression/stretch, vibration, acoustic radiation force, and so forth). ${ }^{20,21}$ The displacement/ velocity vectors and strain/strain-rate tensors are measured simultaneously and can also be used for estimating mechanical properties of such tissues, ${ }^{21}$ such as shear moduli.

For displacement vector measurements, the lateral modulation (LM) methods of Sumi et al ${ }^{11-19}$ are effective. The LM methods can be used only through the use of superposed steered, crossed beams. ${ }^{12-19}$ Specifically, this is the use of superposed multiple steered beams with different steering angles obtained using the multiple transmission method (MTM), ${ }^{20,22}$ or synthesized from a set of received echo data using the multidirectional synthetic aperture method (MDSAM) ${ }^{20,22}$ (see appendix A in Sumi et $\mathrm{al}^{23}$ ). For LM methods, simultaneous or successive transmissions/receptions of a US signal can be used. Multiple transducers can also be used. LM permits echo imaging with almost the same lateral resolution as the axial resolution. ${ }^{15,16}$ For displacement vector measurements, another LM is also performed using the Fraunhofer approximation, as reported by other groups. ${ }^{24-27}$ Their common approach uses an apodization function that displays several continuous peaks. Moreover, this approach performs analogue LM processing twice to obtain multidimensional analytic signals. In contrast, the digital LM approach developed by Sumi et al ${ }^{13}$ produces analytic signals with less processing.

Alternatively, a displacement vector can also be measured by synthesizing axial displacements measured with the highest accuracy possible with regard to the respective beams by using multidimensional displacement measurement methods, including the 1D AM with a multidimensional moving average (MA) $)^{13,22}$ with the MTM or the MDSAM. Thus, this approach also uses new multiple crossed beams (MCBs) with nonsuperimposed or separated beams. ${ }^{13,17,18,20}$ However, LM measurement/imaging can be performed by superimposing the crossed beams. Although 1D measurement methods can also be used for LM, ${ }^{17-19,24-27}$ the MTM, ${ }^{28,29}$ and the $\operatorname{MDSAM}^{30}$ in place of the multidimensional measurement methods, decorrelation of local echo signals occurs because of target displacement in a direction orthogonal to the beams. Although a new demodulation method ${ }^{17-19}$ was developed for LM that uses only digital signal processing, and is different from other demodulation methods, ${ }^{25,27}$ the use of 1D measurement methods, even with the multidimensional MA ${ }^{11-13,22}$ and the multidimensional phase matching, ${ }^{9,10,13}$ results in a lower measurement accuracy than the corresponding multidimensional measurement methods. ${ }^{9}, 10,13,31,32$ 
Recently, Sumi et a $1^{17,18}$ described a steering angle method (ASTA), which is a simpler beamforming method than LM, the MTM, and the MDSAM. ASTA ${ }^{17,18}$ uses only a defined steering angle. All the beamforming methods, including ASTA, can be performed on an arbitrary orthogonal coordinate system. However, ASTA has several advantages over LM, the MTM, and the MDSAM, ${ }^{17,18}$ and fewer calculations are required to complete beamforming than with LM, the MTM, and the MDSAM (see Sumi ${ }^{18}$ and appendix A in Sumi et $\mathrm{a}^{23}$ ).

However, with ASTA, the number of methods available to obtain a displacement vector measurement is limited to the spectra frequency division method (SFDM) $)^{23,33}$ or blockmatching methods ${ }^{17,18}$ such as the MCSPGM, the MCCM, and the MAM and the MDM using a block-matching method (referred to as MAMb ${ }^{17,18}$ and MDMb, ${ }^{17,18}$ respectively). The respective simulations described in Sumi ${ }^{18}$ and Sumi et al ${ }^{23}$ confirmed that, for ASTA with $45^{\circ}$, the MCSPGM and the SFDM failed to yield a higher measurement accuracy than with LM (ie, 100 and 10 times larger SDs, respectively). Basically, for the SFDM with ASTA, the steered beam should be aimed in the direction of the target motion, which increases measurement accuracy in the displacement magnitude measurements, as does the use of 1D displacement measurement methods with ASTA; however, this decreases the accuracy of the displacement angle. ${ }^{23}$ It was also confirmed that echo or coordinate rotation (eg, with no steering) increases the measurement accuracy of the displacement vectors. ${ }^{23}$ With the SFDM, plural multidimensional (two-dimensional [2D] or three-dimensional [3D]) analytic signals are generated by dividing a single-quadrant or single-octant spectra in the corresponding frequency domain to enable the use of the MAM and the MDM, which use multidimensional MAs. With the SFDM and a version of ASTA with nonsteered beamforming (ie, the first version of the MAM or the MDM without LM or beam steering ${ }^{11-13,23,31,33}$ ), the use of laterally, symmetrically divided spectra permits a more accurate measurement than the 1D AM or the 1D DM with a multidimensional MA. ${ }^{31}$ By using a proper apodization, it was possible to gain almost the same accuracy as with $\mathrm{LM}^{23,33}$ (see discussions and conclusions section).

Alternatively, because only a single-quadrant or singleoctant spectra is obtained using ASTA, assuming there is no displacement in the axial direction, lateral displacement measurements ${ }^{17,18}$ can be made using the $\mathrm{MAM}^{11-13}$ and the $\mathrm{MDM}^{11-13}$ with a multidimensional MA. In addition to the application of multidimensional methods, simpler lateral displacement measurement methods have also been developed; for instance, the mirror-setting method, ${ }^{17,18}$ the lateral Doppler techniques (lateral AM or DM, which divide a multidimensional MA instantaneous phase change by a multidimensional MA lateral frequency), ${ }^{17,18,23}$ and others. For the two lateral measurement methods, the lateral coordinate axis must be set in the direction of the target lateral motion by rotating the coordinate system analytically or approximately (interpolation) during beamforming, or after beamforming in a spatial or frequency domain. ${ }^{17,18,23}$ These methods are expected to be useful for measuring blood flow in vessels running parallel to the body surface (eg, in the carotid artery). Thus, ASTA will permit the use of simpler manual techniques to obtain measurements for medical doctors or clinical staff personnel than conventional Doppler measurement techniques. However, in order to increase measurement accuracy, a sensor for the mechanical steering angle and an automatic detector for blood vessels are required.

However, except for LM, the aforementioned measurement methods using beamforming methods such as MCB, ASTA, conventional steering (eg, secta, radial scan, and so forth), nonsteering, and others have not dealt with problems in acquiring highly accurate data from beam directions generated in practice. ${ }^{34}$ In real-world applications, the beam direction generated differs from the designed one because of the existence of directivities of transmission and reception apertures. Moreover, the beam direction changes in different positions because of the effects of scattering, reflection, and attenuation. Thus, the beam direction also suffers from frequency modulations. As shown in this report, this decreases the measurement accuracy of displacement measurements ${ }^{34}$ (eg, measurements of displacement vectors and of axial, lateral, or radial displacements). However, by estimating the respective center of the beams (eg, the center of the spectra), the beam direction can be calculated, as shown in Sumi. ${ }^{18}$ Such calculations can also be applied to local echo data to yield spatial resolution in the beam direction or beam angle (BA) data. ${ }^{34}$ However, estimating the instantaneous frequencies in all directions (ie, when done with the MAM or the MDM) also permits the calculation of the BA with a high spatial resolution. ${ }^{18,34}$ In the past, the BA data used in displacement measurements were set at values for performing analogue or digital beamforming to generate the beams designed (ie, determined by delays in the respective US elements) (see Appendix 1 for application to the conventional 1D AM ${ }^{3}$ or Sumi et al's ${ }^{13,19,22,31}$ developed 1D AM or 1D DM with a multidimensional MA).

In this report, new displacement measurement methods that use highly accurate BA data with a spatial resolution are investigated..$^{34}$ The new methods are referred to as BA methods or methods used in a BA approach. With all the 
beamforming methods, except for LM, the new methods can be used for all displacement measurements. For instance, for displacement vector measurements, the BA approach is applied to MCB at each point of interest. The use of accurate motion/deformation direction data obtained for nonsteered beamforming or ASTA also permits accurate measurement of displacements in an arbitrary direction (ie, of an arbitrary directional displacement such as displacements in axial and lateral directions) except in a direction orthogonal to the beam direction. In these cases, the most popular 1D AM, ${ }^{3}$ and Sumi et al's ${ }^{13,19,22,31}$ developed 1DAM, 1D DM or the SFDM ${ }^{23,31}$ can be used. However, in all of the measurement methods, the MA to be calculated is multidimensionally similar to the MAM or the MDM. ${ }^{13,31}$ In conventional measurements using MCB or beam steering, echo rotation is performed to adjust the beam direction to the axial direction of a coordinate system (see Appendix 1, in which the conventional 1D AM is referred to as the 1D AMr, which is the 1D AM plus a global echo rotation of $\mathrm{r}$ ). This is a conventional $1 \mathrm{D} \mathrm{AM}$ with a rotation of the echo data frame or ROI or coordinate system with inaccurate BA data. The new BA method permits the same measurements without any rotation processing of echo data. Even though an accurately measured beam direction is used, the local echo rotation yields a low measurement accuracy, particularly when using an interpolation method such as a linear interpolation instead of a phase rotation method in a frequency domain ${ }^{9,10,13}$ with a phase-matching method. For comparison, such methods using a local echo rotation are referred to as a BAr method (BA + local rotation). The rotation processing also makes it impossible to achieve measurements in real time, as will be explained.

The effectiveness of the BA approach is confirmed for axial (nonsteered), lateral (ASTA), and vector (MCB) measurements in agar phantom experiments. Generally, steered spherical focusing beams are used for both transmission and reception. In Appendix 4, measurement results are also presented for rapid scanning beamforming, ${ }^{13,15,16,23}$ such as beamforming using one or plural, steered or nonsteered plane wave transmissions, and steered or nonsteered spherical focusing beam receptions.

\section{New displacement measurements using measured BA data} Displacement measurements in a generated
beam direction and in an arbitrary direction A displacement in a beam's direction can be accurately obtained when scanning a region of interest (ROI) using arbitrary beamforming with an arbitrary transducer (eg, linear, convex, and so forth), with nonsteered, steered with a variable steering angle (eg, secta, arc, circular, radial scan, and so forth), or with a defined ASTA, or with other methods, at each point of interest, with a generated beam direction. ${ }^{34}$ The beam direction can be calculated at each point of interest using the first moment of the local spectra obtained for local echo data centered on the point of interest, or from the instantaneous frequencies of the respective directions obtained using a multidimensional MA with the MAM or the $\mathrm{MDM}^{11-13}$ (see sections II-B and II-C in Sumi ${ }^{13}$ ).

For instance, when dealing with a 2D ROI expressed by a Cartesian coordinate system (ie, when using a 1D linear array transducer), if the first moments or instantaneous frequencies are expressed as $\left(f_{x}, f_{y}\right)$, a BA $\alpha$ describing the beam direction at the point of interest $(x, y)$ in Figure 1 can be calculated by

$$
\alpha=\tan ^{-1}\left(f_{y} / f_{x}\right)
$$

An approach that uses the spatial resolution of the generated BA is referred to as a BA approach. Initially, the BA approach is applied with the conventional 1D AM or 1D DM using global echo rotation. For instance, from the conventional 1D AM approach with inaccurate BA data (see Appendix 1, in which the 1D AM is referred to as 1D AMr, ie, conventional 1D AM with a rotation of the echo data frame or ROI or a coordinate system rotation), two new 1D AMs (the 1D AMBA and the 1D AMBAr) can be obtained as follows.

\section{The ID AMBA (ID AM + BA method)}

With the conventional 1D AM, the displacement $d(x, y)$ at the point of interest $(x, y)$ in the beam direction shown in Figure 2 (the BA $\alpha$ obtained by Equation 1) can be obtained simply by dividing the instantaneous phase change $\Delta \theta(x, y)$ between two frames by the frequency $\sqrt{\left(f_{x}^{2}+f_{y}^{2}\right)}$ in the beam direction as follows:

$$
d(x, y)=\Delta \theta(x, y) / \sqrt{\left(f_{x}^{2}+f_{y}^{2}\right)}
$$

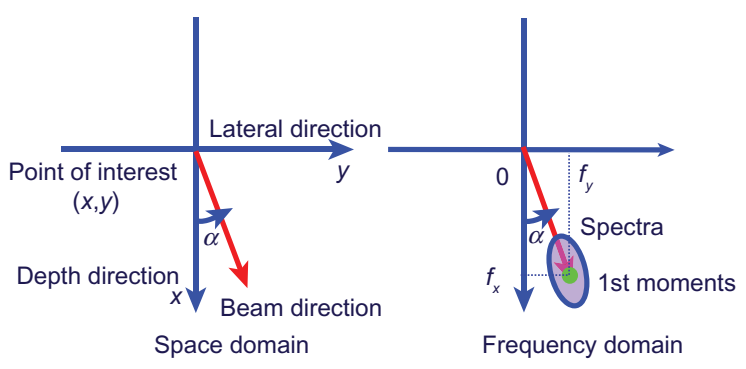

Figure I For a two-dimensional case, this is a schematic of the beam direction and generated beam angle $\alpha$ and the first moments of the local spectra or instantaneous frequencies $\left(f_{x}, f_{y}\right)$ at a point of interest $(x, y)$. 


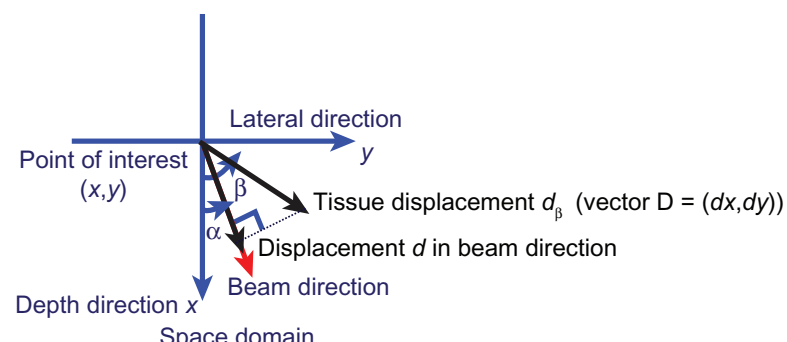

Space domain

Figure 2 For a two-dimensional case, this is a schematic of the displacement $d$ in the generated beam direction (beam angle, $\alpha$ ) and tissue displacement $d_{\beta}$ (angle, $\beta$ ) at a point of interest $(x, y)$.

Here, the instantaneous phase change $\Delta \theta(x, y)$ is calculated from the multidimensional MA used in the MAM or the $\mathrm{MDM}^{11-13}$ (see sections II-B and II-C in Sumi ${ }^{13}$ ) in a manner similar to the instantaneous frequencies $\left(f_{x}, f_{y}\right){ }^{11,13} \mathrm{Next}$, $\Delta \theta(x, y)$ calculated in such a manner expresses the change in the instantaneous phase of the generated beam direction that occurs because of target motion at a point between the two frames. The calculation of $\Delta \theta(x, y)$ with the multidimensional MA yields the new measurement. As mentioned, $\left(f_{x}, f_{y}\right)$ can also be obtained as the first moment of local spectra.

For instance, if the direction of tissue displacement $d_{\beta}(x, y)$ can be detected geometrically, such as from a corresponding US image or from other images (eg, the tissue displacement angle $\beta$ shown in Figure 2), the tissue displacement $d_{\beta}(x, y)$ can be calculated as follows: ${ }^{34}$

$$
\begin{aligned}
d_{\beta}(x, y) & =d(x, y) / \cos (\beta-\alpha) \\
& =\Delta \theta(x, y) /\left(f_{x} \cos \beta+f_{y} \sin \beta\right) .
\end{aligned}
$$

Thus, being different from the conventional 1D AMr (Appendix 1), without any rotation of echo data, the 1D AMBA yields a displacement $d_{\beta}(x, y)$ measurement in an arbitrary direction (tissue displacement angle, $\beta$ ) in real time except in the direction orthogonal to the beam direction (BA, $\alpha$ ). That is, $d_{\beta}(x, y)$ cannot be calculated when $|\beta-\alpha|=90^{\circ}$. As shown by Equation $3^{\prime}, d_{\beta}(x, y)$ can also be calculated directly using $\Delta \theta(x, y),\left(f_{x}, f_{y}\right)$, and $\beta$ without the calculation of BA $\alpha$ (Equation 1) and the use of Equation 2.

The 1D AMBA using Equations 1-3, or 3' can be used for an arbitrary BA $\alpha$. For instance, for an arbitrarily shaped aperture, such a measurement can also be performed with no steering $\left(\alpha=0^{\circ}\right)$, except for the lateral displacement method with an angle $\beta=90^{\circ}$. Generally, the lateral frequency $f_{y}$ (ie, fluctuations) is not considered in such a case and is not used for calculations. However, such a multidimensional MA has also been used in 1D displacement measurement methods such as the 1D AM where $f_{y}=0$ in Equations 1-3, or 3' (Equations A3 and 3' in Appendix 1; see Kasai et al, ${ }^{3}$
Sumi, ${ }^{13,22}$ Sumi and Shimizu, ${ }^{19}$ and Sumi and Ebisawa $\left.{ }^{31}\right)$. However, when the lateral generated frequency fluctuation $f_{v}$ or the tissue displacement angle $\beta$ is large, measurement errors increase.

If beam steering or ASTA is performed electrically or mechanically $\left(\alpha \neq 0^{\circ}\right)$, beams should be aimed in the direction of the target displacement. For instance, when blood flows in a direction parallel to the body surface, such as in the carotid artery, $\beta \approx 90^{\circ}$ in Equations 3 or $3^{\prime}$, and the tissue displacement $d_{\beta}(x, y)$ can be calculated as follows:

$$
\begin{aligned}
d_{\beta}(x, y) & =d(x, y) / \sin \alpha \\
& =\Delta \theta(x, y) / f_{y} .
\end{aligned}
$$

This is because a displacement in the axial direction is $d x=0$ or $d x \approx 0, d_{\beta} \approx d y$ (this is a lateral displacement). Thus, in this case, the 1D AMBA using Equations 1-3 or $3^{\prime}$ is equivalent to Sumi et al's ${ }^{17,18,34}$ previously described lateral Doppler techniques (lateral AM or DM). However, it is worth noting that, unlike the lateral DM, the 1D AMBA does not require a lateral axis coordinate system oriented in the same direction as the target lateral displacement; that is, a rotation of the echo data or of a coordinate system is not required.

Similarly, for a 3D ROI, ${ }^{34}$ a displacement $d(x, y, z)$ in the beam direction can be measured, and a tissue displacement $d_{\beta}(x, y, z)$ in an arbitrary direction can be accurately measured (see Appendix 2). The first moments or instantaneous frequencies in three orthogonal directions $\left(f_{x}, f_{y}, f_{z}\right)$ or rotational and polar angles ( $\varphi$ and $\theta$ ) are used.

As an alternative to the 1D AM, the 1D DM can also be used as a 1D displacement measurement method (Sumi, ${ }^{13,22}$ Sumi and Shimizu, ${ }^{19}$ and Sumi and Ebisawa ${ }^{31}$ ). Correspondingly, based on the 1D DM and the 1D DMr, the 1D DMBA (1D DM + BA method) and the 1D DMBAr (1D DM + BAr method) can also be used (see Appendix 1). Although the instantaneous phase change and instantaneous frequencies are calculated in a different manner, as in the 1D AM, a multidimensional MA is also used for the calculations (see section II-C in Sumi ${ }^{13}$ ). However, in this report, the 1D DM approach is not evaluated.

For comparisons with the 1D AMBA and the conventional 1D AMr (Appendix 1), the 1D AMBAr is compared (1D AM with local BA rotation, or BA $+r$ ) using $B A \alpha$ (Equation 1). In contrast to the conventional 1D AMr using global echo rotation with inaccurate, averaged BA data (see Appendix 1), with the 1D AMBAr, the echo data are locally rotated at each point of interest using accurately measured BA data for $\alpha$, so that the beam direction corresponds to the axial direction (ie, a local echo rotation is performed) (Figure 3). 

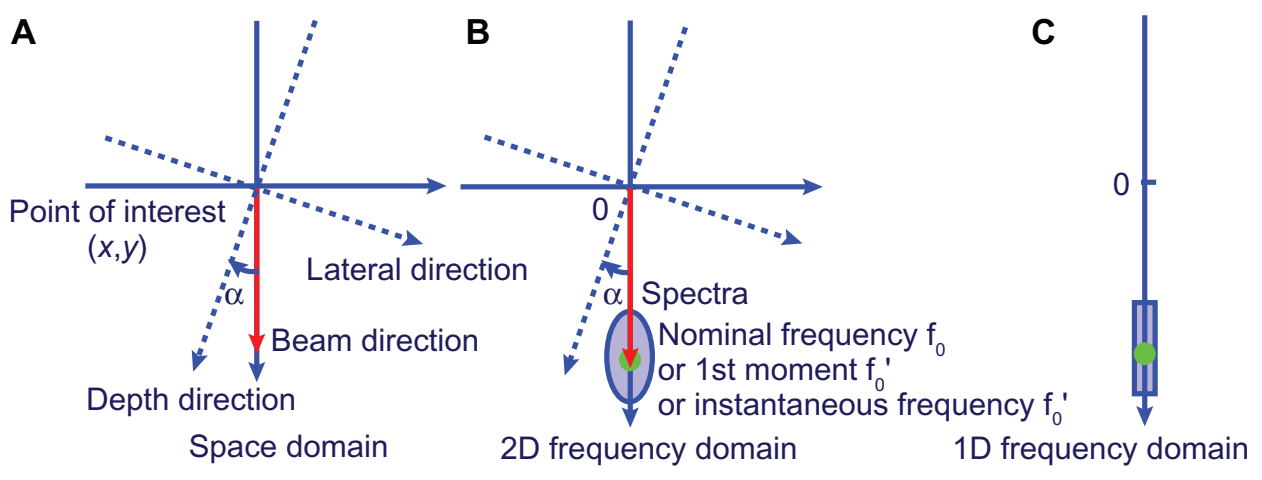

Figure 3 For a two-dimensional (2D) case, this is a schematic of the (A) space and (B) frequency domains after a rotation with angle $-\alpha$ at a point of interest $(x, y)$. (C) Schematic for the one-dimensional (ID) frequency domain.

\section{The ID AMBAr (ID AM + BAr method)}

When rotating echo data for displacement measurements in a beam direction based on the conventional 1D AMr (see Appendix 1), accurate data for the BA $\alpha$ should also be used (Figure 3). ${ }^{34}$ With the conventional 1D AMr, the BA data for $\alpha$ provides inaccurate values (they are described as $\alpha^{\prime}$ in Appendix 1), because the analogue or digital beamforming used to generate the designed beam is defined by delays assigned to the respective US elements, rather than by obtaining accurate BA data for $\alpha$ expressed in Equation 1. In the authors' case, ${ }^{23}$ for global echo rotation, in practice, the global BA has been calculated with Equation 1 using the first moments $\left(f_{x}^{\prime}, f_{y}^{\prime}\right)$ of the global spectra (see also Appendix 1). ${ }^{23}$

In contrast, the $1 \mathrm{D}$ AMBAr performs a local rotation of echo data at each point of interest $(x, y)$ using Equation 1 in a spatial or frequency domain, as shown in Figure 3. In such a 2D case, the Fourier transform is $2 \mathrm{D}$ (Figure $3 \mathrm{~A}$ and $\mathrm{B}$ ) or 1D (Figure 3C). Although the global rotation of echo data using the first moments of the global spectra ${ }^{23}$ also yields a higher measurement accuracy than a conventional global echo rotation, the achievable accuracy is lower than what can be obtained by a local rotation (eg, 1D AMBAr), as will be shown shortly. However, 1D AMBAr measurements cannot be accomplished in real time, owing to the large number of calculations needed for local rotations over an ROI, and they yield less accurate measurements than the 1D AMBA.

For rotation in a $3 \mathrm{D}$ case, a rotational angle $\varphi$ and a polar angle $\theta$ are calculated using three frequencies $\left(f_{x}, f_{y}, f_{z}\right)$ that are accurately measured in three orthogonal directions (see Appendix 2) and used in a manner similar to that for the $2 \mathrm{D}$ case.

For Sumi et al's ${ }^{23,33}$ previously described SFDM, accurate BA data for $\alpha$, the rotational angle data for $\varphi$, and the polar angle data for $\theta$ (ie, steering angles for 2D and 3D ROIs) can be used to increase measurement accuracy (ie, by deterieing a direction for the spectra division in addition to performing angle corrections using a tissue displacement angle). From the respective divided spectra, equations are obtained for the MAM or the MDM by synthesizing instantaneous frequencies and an instantaneous phase change. As already mentioned, for a nonsteered case, the SFDM is the first version of the MAM without LM or any steering. ${ }^{13,23,31,33}$ For 2D and 3D ROIs, a straight line or a flat plane can be used to divide spectra. Sumi et $\mathrm{al}^{23}$ have also described some other division methods. For instance, a curved line or a curved plane can also be used for the spectra division. When obtaining more equations than the number of unknown displacement components with the SFDM, the least squares method can be used. However, these methods were not used in this report.

In addition to the original SFDM, Sumi et $\mathrm{al}^{23}$ have developed two other versions of the method. That is, when using the original SFDM version, displacement in a steeredbeam direction can be synthesized by measuring a displacement vector without echo rotation, whereas when using the SFDM1 (version 1, referred to as SFDM1r in this report), the displacement in the steered-beam direction can be obtained by setting the generated beams in the axial direction with echo rotation as with the conventional 1D AMr; and when using the SFDM2 (version 2), the lateral displacement is directly obtained as a lateral displacement component of the measured displacement vector after an echo rotation similar to the one used with the lateral Doppler techniques (ie, the direction of the lateral axis is set in the direction of the dominant lateral displacement). With the SFDM2, BA data is not used, so neither the BA nor the BAr method is used with the SFDM2. Alternatively, the SFDM and the SFDM1 can be improved by using accurate BA data and are referred to as the SFDMBA (the SFDM + BA method) ${ }^{34}$ and the SFDM1BAr 
(the SFDM1r + BAr method) ${ }^{34}$ respectively. The SFDMBA and the SFDM1BAr are described as follows.

\section{The SFDMBA (SFDM + BA method)}

On the basis of the original SFDM, ${ }^{23}$ the single-quadrant or single-octant spectra obtained with ASTA is divided to obtain simultaneous equations (the MAM or the MDM) $)^{11-13}$ for the unknown displacement vector $D(x, y)$ components (Figures 2 and 4). From the measured displacement vector, the displacement $d(x, y)$ or $d(x, y, z)$ in the beam direction can be calculated with a high degree of accuracy. The BAs are measured, and Equation 1 expresses $\alpha$ for a 2D case; Equation B1 in Appendix 2 expresses the rotational angle $\varphi$ and the polar angle $\theta$ for a $3 \mathrm{D}$ case. Tissue displacement $d_{\beta}(x, y)$ or $d_{\beta}(x, y, z)$ is also obtained with high degrees of accuracy such as by using Equation 3. In a manner similar to that for the 1D AMBA, this measurement can be accomplished with no echo rotation. However, the number of calculations required is greater than for the 1D AMBA because of the use of the MAM or the MDM. In this report, the MAM is used.

\section{The SFDMIBAr (SFDMI + BAr method)}

As already mentioned, the original $\mathrm{SFDM}^{23}$ is referred to as SFDM1r in this report. It uses a global echo rotation based on axial displacement measurements ${ }^{13,31}$ obtained with the original MAM or the MDM without LM or beam steering. For a nonsteering case (ie, a version of ASTA), ${ }^{13,31}$ the SFDM yields a higher measurement accuracy than conventional methods such as the 1D AM. This is because of synthesizing a lateral instantaneous frequency and a lateral instantaneous phase for laterally large bandwidth spectra echo data. Using a proper apodization, almost the same accuracy is achieved as when using the MAM with LM. ${ }^{23,33}$ Moreover, for the SFDM with ASTA with a nonzero steering angle, an echo rotation

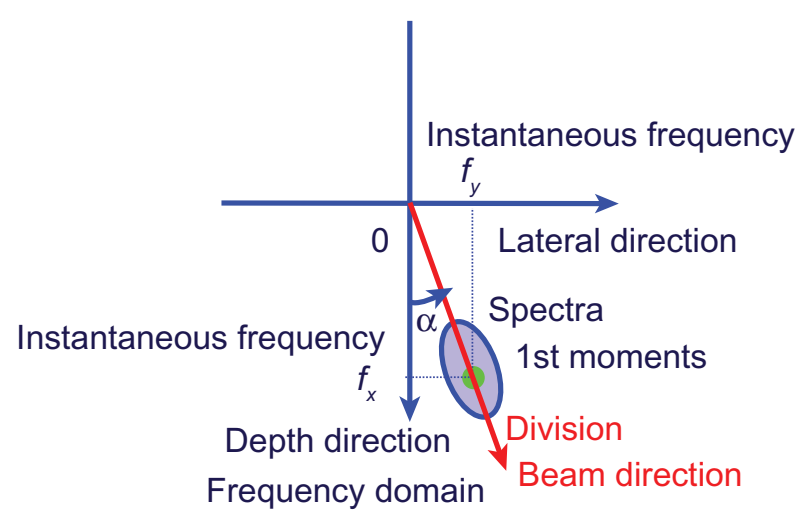

Figure 4 For a two-dimensional case, this is a schematic of spectra frequency division using the first moments or instantaneous frequencies $\left(f_{x}, f_{y}\right)$. for such a nonsteered condition is realized, which increases measurement accuracy. ${ }^{23}$ The degree of improvement depends on the lateral bandwidth of the steered beam.

Correspondingly, with the SFDM1BAr using local echo rotation, the SFDM (see Figure 4) is used for measuring displacements $d(x, y)$ or $d(x, y, z)$ in the beam direction after an echo rotation with a negative value of the accurately measured BA (Equation 1) - that is, $-\alpha$ (Figure 3B) or $-\varphi$ and $-\theta$ (Appendix 1). Thus, a tissue displacement $d_{\beta}(x, y)$ or $d_{\beta}(x, y, z)$ is obtained from $d(x, y)$ or $d(x, y, z)$ such as by using Equation 3. However, such echo rotations over the ROI produce no real-time measurements.

For arbitrary orthogonal coordinate systems including arbitrary orthogonal curvilinear coordinates, all the new BA measurements can be performed. This also applies for the MCB methods with a BA approach, as described next.

\section{Displacement vector measurements using $\mathrm{MCB}$}

For MCB, the BA approach - the 1D AMBA, the 1D AMBAr, the SFDMBA, and the SFDM1BAr - can also be used. ${ }^{34}$ At each point of interest, these methods can be applied to the respective beams generated for measuring displacements in the corresponding beam directions with high accuracy. By synthesizing the displacements measured in the respective beam directions, a displacement vector in an arbitrary direction can be measured with high accuracy.

For instance, for a 2D displacement vector measurement at a point of interest $(x, y)$, as shown in Figure 5, two beams with different directions must be crossed at least at the point $(x, y)$. When the displacements $d_{1}$ and $d_{2}$ measured in the two beam directions are expressed using the BAs $\alpha_{1}$ and $\alpha_{2}$ shown in Figure 5, the tissue displacement vector $D=(d x, d y)$ can be measured by solving the following simultaneous equations:

$$
\begin{aligned}
& \cos \alpha_{1} d x+\sin \alpha_{1} d y=d_{1} \\
& \cos \alpha_{2} d x-\sin \alpha_{2} d y=d_{2} .
\end{aligned}
$$

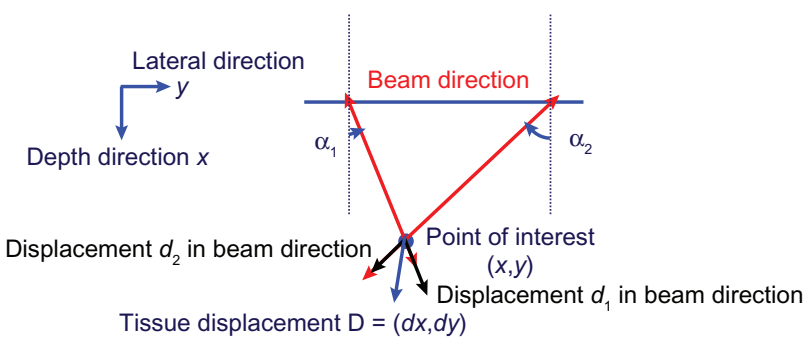

Figure 5 For a two-dimensional case, when using multiple crossed beams, the tissue displacement $D$, and displacements $d_{1}$ and $d_{2}$ in the respective beam directions are shown with beam angles $\alpha_{1}$ and $\alpha_{2}$ generated at a point of interest $(x, y)$. 
That is, the displacements are solved as

$$
\begin{aligned}
& d x=\left(\sin \alpha_{2} d_{1}+\sin \alpha_{1} d_{2}\right) /\left(\sin \alpha_{2} \cos \alpha_{1}+\sin \alpha_{1} \cos \alpha_{2}\right) \\
& d y=\left(\cos \alpha_{2} d_{1}-\cos \alpha_{1} d_{2}\right) /\left(\sin \alpha_{2} \cos \alpha_{1}+\sin \alpha_{1} \cos \alpha_{2}\right) .
\end{aligned}
$$

Generally, if obstacles (eg, bone) are not present, laterally symmetric beams can be used $\left(\alpha_{1}=\alpha_{2}\right){ }^{23}$

In this situation, suppose that when applying LM with the MAM or the MDM to the same superimposed or separated beams at the point of interest $(x, y)$, an equation for the displacement components $d x$ and $d y$ is obtained for the beam with BA $\alpha_{1}$ as follows:

$$
f_{x 1} d x+f_{y 1} d y=\Delta \theta_{1}
$$

where $\Delta \theta_{1}$ is an instantaneous phase change at the point $(x, y)$ between two frames, and $f_{x 1}$ and $f_{y 1}$ are the respective instantaneous frequencies at the same point $(x, y)$ in the axial and lateral directions, and have a relationship to the $\mathrm{BA} \alpha_{1}$ as follows:

$$
\alpha_{1}=\tan ^{-1}\left(f_{y 1} / f_{x 1}\right)
$$

Division of Equation 7 by $\sqrt{\left(f_{x 1}^{2}+f_{y 1}^{2}\right)}$ yields the first of the simultaneous equations that make Equation 5; that is,

$$
\left(f_{x 1} / \sqrt{\left(f_{x 1}^{2}+f_{y 1}^{2}\right)}\right) d x+\left(f_{y 1} / \sqrt{\left(f_{x 1}^{2}+f_{y 1}^{2}\right)}\right) d y=d_{1} .
$$

Similarly, regarding another beam with BA $\alpha_{2}$, the second of the simultaneous equations that make Equation 5 is obtained. Thus, the application of accurate BA data to MCB yields LM. For measurement of a 3D displacement vector, three beams with different directions must be crossed at least at the measured point (consider the root of summation of three squared instantaneous frequencies, rotational angle, and polar angle, as shown in Appendix 3).

When obtaining more equations than the number of unknown displacement components, the least-squares method is used similarly to that in the case of LM. ${ }^{11-13}$ The beams that can possibly be used include a nonsteered beam similar to the case for LM when considering the simultaneous use of conventional nonsteered B-mode imaging.

\section{Multidimensional phase matching performed with the use of BA data}

Multidimensional phase matching permits manual handling of a US transducer for axial strain imaging or strain tensor/ shear modulus measurement/imaging. 9,10,13,15,16,32,35 The phase matching prevents the occurrence of aliasing because of a large tissue motion during successive frames used for motion measurements, and it also increases an incoherence between paired local or windowed echo signals to be processed. ${ }^{9,10,13,32}$ That is, the MCCM ${ }^{7,8}$ or the MCSPGM ${ }^{9,10}$ is used for measuring a large tissue motion vector. ${ }^{13,32,35}$ The measurement can also be performed with spatially thinned-out echo data. ${ }^{13,20}$ Because a coarse estimate of a displacement vector is obtained by such a measurement, by spatially shifting the corresponding local windowed echo data or a search region echo data with the coarse estimate, multidimensional phase matching is achieved. Although the phase matching can also be performed in a Fourier domain by a phase rotation of the echo data, the calculation makes real-time measurement/imaging difficult. Because the MCSPGM yields an analogue estimate with fewer calculations than the MCCM, when using the MCSPGM for the coarse estimate, the measured displacement data may be rounded off over the multiple sampling intervals. After the phase matching, a fine estimate is performed using the new BA approach described earlier for a displacement measurement in an arbitrary direction or a displacement vector measurement.

In the global echo rotation case (ie, the $\mathrm{r}$ method), phase matching is performed simply before or after the global rotation. Alternatively, phase matching can be performed in three ways when performing a local echo rotation - that is, when using the BAr methods (1D AMBAr or SFDM1BAr). The timing of the phase matching to be performed can be as follows: (1) after completing an echo rotation, coarse and fine estimates are successively performed with phase matching on the rotated echo data; (2) after completing a coarse estimate and phase matching, the echo data are rotated and used for fine measurements; (3) a coarse measurement is performed before echo rotation, and the coarse estimate obtained is re-expressed on the new coordinate obtained with echo rotation and is used for both phase matching and fine measurements.

On the basis of the estimate for the magnitude of a target displacement, the size of a local region (a rectangle, circle, cube, or sphere) and a search region with at least a twofold length of the local region are defined and used at first, and a region of the same size or larger than the search region is rotated using an accurately measured BA. For fine measurements, a small local region (and/or search region) can be used. ${ }^{10,13}$

As noted earlier, when local echo rotations are performed on respective points of interest, a real-time measurement cannot be achieved. Moreover, MCB using accurate BA 
data requires more calculation time than LM. This is because MCB performs the phase matching and/or echo rotation on the respective steered beams, although measurement processing can decrease if the coarse estimation is performed on the superimposed crossed beams in a similar way to that of LM. Moreover, although LM requires a multidimensional discrete Fourier transform (DFT) to be performed once, MCB requires the same plural number of DFTs as steered beams. Generally, a fast Fourier transform is performed for the DFT.

\section{Agar phantom experiments}

For testing these new methods, three experiments were performed that were distinguished by beamforming and the target measurements: (1) axial displacement/strain measurements using a nonsteered beam; (2) lateral displacement/ strain measurements using ASTA; and (3) displacement vector/strain tensor measurements using $\mathrm{MCB}$.

\section{Axial displacement/strain measurements using a nonsteered beam}

Nonsteered beamforming was performed using a linear array transducer. The agar phantom used was the same as that used in Sumi and Ebisawa. ${ }^{31}$ The rectangular ROI with the dimensions of 23.8 (axial) $\times 19.4$ (lateral) $\mathrm{mm}$ was set at a depth of $3.2 \mathrm{~mm}$. A central circular region (a cylindrical inclusion with a diameter of $15 \mathrm{~mm}$ and a depth of $15 \mathrm{~mm}$ ) had a larger shear modulus than the surrounding region: a relative value of $3.33\left(2.96\right.$ vs $\left.0.89 \times 10^{6} \mathrm{~N} / \mathrm{m}^{2}\right)$. The agar phantom was compressed in the depth (axial) direction. The nominal US frequency of the transducer was $7.5 \mathrm{MHz}$. The agar phantom was compressed with global strains $0.7 \%$ and $1.5 \%$ in the axial direction twice.

For the global axial compressions (compressional strains) of $0.7 \%$ and $1.5 \%$, Figure 6 shows a depth (axial) position vs the means and standard deviations (SDs) of BAs measured at each depth using the first moments of local spectra (echo data with a local region with dimensions of $1.6 \times 3.2 \mathrm{~mm}^{2}$ ). As has been shown, the BAs change in the depth direction. However, almost the same results are obtained when independent of the compression force. The absolute means (biases) and SDs of the BAs generated are less than $0.5^{\circ}$ and $1.0^{\circ}$, respectively (BAs $<1.5^{\circ}$ ). Thus, the lateral frequencies $\left(f_{y}\right)$ generated are less than $0.39 \mathrm{MHz}$, or $5 \%$ of the axial frequencies $\left(f_{x}\right)$. The depth-dependent changes of the means and SDs are relatively drastic and moderate, respectively. It is also worth noting that the SDs are larger than the means. Obviously, the nonzero BAs are caused by the spatially variant US properties of the target agar phantom (reflection, scattering, attenuation, and frequency modulation). As will be shown shortly, the measurement results are more stable when using the BA data $\left(1.6 \times 3.2 \mathrm{~mm}^{2}\right)$

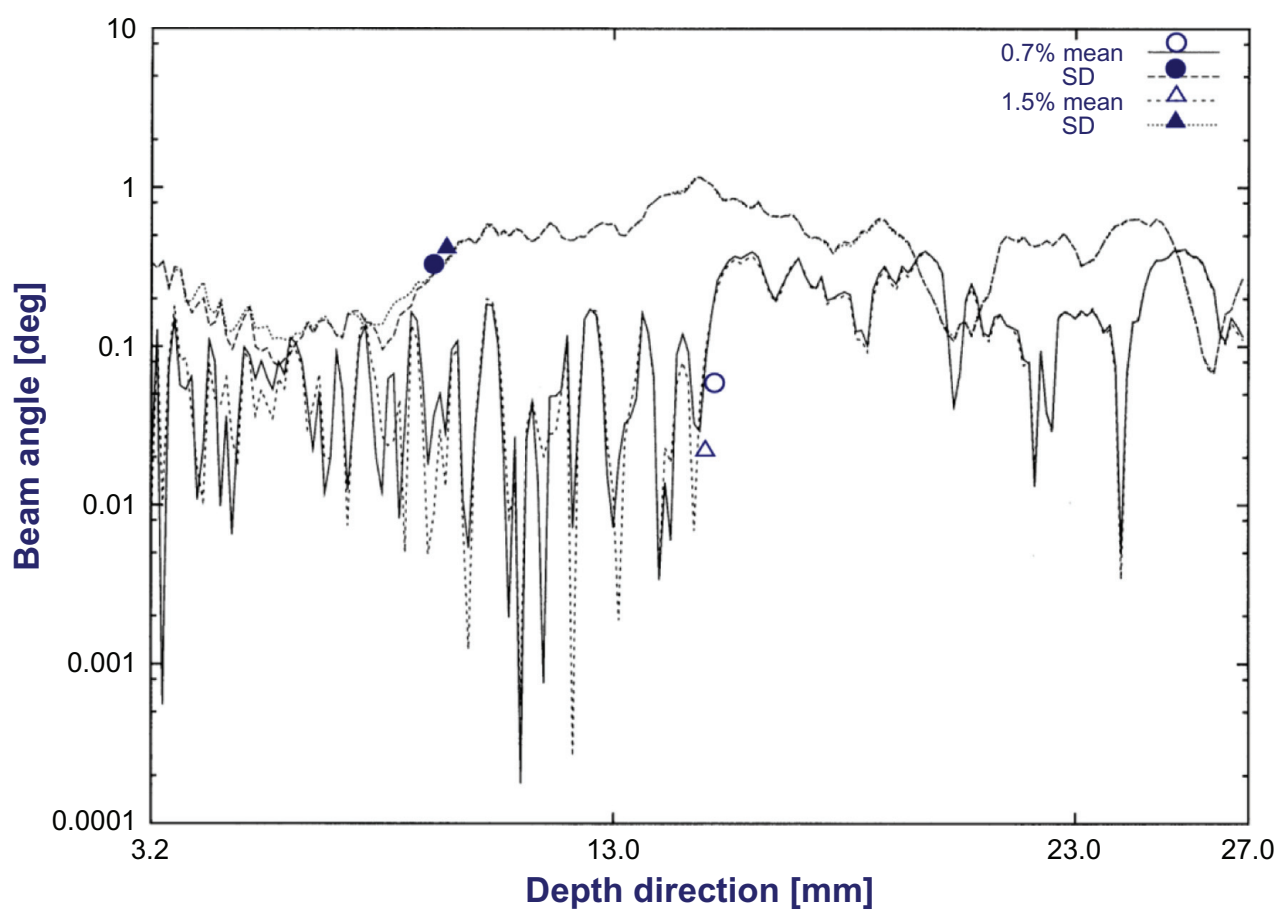

Figure 6 For an agar phantom axially compressed with respective global strains, $0.7 \%$ and $1.5 \%$, the depth vs means and standard deviations (SDs) of the beam angles are shown. These were measured using the first moments of local spectra with spatial resolution $1.6 \times 3.2 \mathrm{~mm}^{2}$.

Abbreviation: deg, degrees. 
than when using the first moments of the spectra of local echo data with a large size $\left(3.2 \times 6.4 \mathrm{~mm}^{2}\right)$ and MA instantaneous frequencies with a small size $\left(0.5 \times 0.6 \mathrm{~mm}^{2}\right)$.

For a large global axial compression (1.5\%), Figure 7A shows the means and SDs of the measured BAs using the first moments of the local echo data spectra with the same small size as that shown in Figure $6\left(1.6 \times 3.2 \mathrm{~mm}^{2}\right)$ and with the large size $\left(3.2 \times 6.4 \mathrm{~mm}^{2}\right)$, and instantaneous MA frequencies with $0.5 \times 0.6 \mathrm{~mm}^{2}$. The order of the means of the measured BAs is as follows: first moments with $1.6 \times 3.2 \mathrm{~mm}^{2}<3.2 \times 6.4 \mathrm{~mm}^{2}<$ instantaneous frequencies $\left(0.5 \times 0.6 \mathrm{~mm}^{2}\right)$. The order of the corresponding SDs is as follows: first moments with $3.2 \times 6.4 \mathrm{~mm}^{2}<1.6 \times 3.2 \mathrm{~mm}^{2}<$ instantaneous frequencies $\left(0.5 \times 0.6 \mathrm{~mm}^{2}\right)$. In addition, Figure $7 \mathrm{~B}$ shows the corresponding grayscale images of axial displacements and strains measured using the three BA data sets together with those measured using the original 2D AM method with no steering (ie, the SFDM using global spectra divided with plus and minus lateral frequencies) and the conventional 1D AM with no BA correction. In the grayscale images (Figure 7B), the means and SDs evaluated at the central circular region of the inclusion are depicted (the diameters are 5.0 and $6.4 \mathrm{~mm}$ for the displacement and strain evaluations, respectively).

In particular, for the strain measurements, the use of the first moments with $1.6 \times 3.2 \mathrm{~mm}^{2}$ yields the highest accuracy and stability of the $\mathrm{BA}$ methods (the $\mathrm{SD}$ for the strain is $0.141 \%$ (the smallest); $0.148 \%$ with the first moments with $3.2 \times 6.4 \mathrm{~mm}^{2}$; and $0.153 \%$ with the instantaneous frequencies). This is because the use of a large local size $\left(3.2 \times 6.4 \mathrm{~mm}^{2}\right)$ yields inaccurate BA data due to a low spatial resolution (ie, a larger mean and a smaller SD than those for a small local size $\left[1.6 \times 3.2 \mathrm{~mm}^{2}\right]$ ). The unstable BA data are obtained using the instantaneous frequencies with a spatial resolution that is too high (ie, the largest mean and SD of the BA methods). Also worth noting is that, in particular, the change of the SD of a BA measured with $1.6 \times 3.2 \mathrm{~mm}^{2}$ is the most moderate of all. Although the $f_{y}$ values generated are small, a significant improvement in measurement stability is confirmed (the SD of the axial strains is $0.141 \%$ using the optimal BA method vs $0.148 \%$ using the 1D AM with no BA correction; however, it is $0.128 \%$ (the smallest) with the 2D AM). Such an improvement cannot be confirmed for the displacement measurements (SDs of 9.17 [2D AM], 9.49 [1D AM with no BA correction]; 9.48 [first moments with $1.6 \times 3.2 \mathrm{~mm}^{2}$; 9.77 [first moments with $3.2 \times 6.4 \mathrm{~mm}^{2}$ ] $9.49 \times 10^{-3} \mathrm{~mm}$ [instantaneous frequencies]).

\section{Lateral displacement/strain measurements using ASTA}

ASTA was next performed using a linear array transducer. For ASTA, new methods based on the BA approach (BA, BAr, SFDMBA, and SFDM1BAr) were compared with the corresponding methods with neither the BA method nor the BAr method (ie, conventional 1D AM with global rotation [1D AMr], SFDM, and SFDM1r). The agar phantom used was the same one described in Sumi et al, ${ }^{15,23}$ Sumi and Tanuma, ${ }^{16}$ Sumi and Shimizu. ${ }^{19}$ The rectangular ROI with dimensions of 13.7 (axial) $\times 13.2$ (lateral) $\mathrm{mm}$ was set at the depth $12.2 \mathrm{~mm}$, of which a central circular region (a cylindrical inclusion with a diameter of $10 \mathrm{~mm}$ and a depth of $19 \mathrm{~mm}$ ) had a larger shear modulus than the surrounding region (a relative value of 3.29 [2.63 vs $\left.0.80 \times 10^{6} \mathrm{~N} / \mathrm{m}^{2}\right]$ ). The agar phantom was compressed in the lateral direction. Synthetic aperture data were acquired using a nominal US frequency of 7.5 MHz. For transmission and reception apodizations for ASTA, parabolic functions were used. ${ }^{15,16}$ ASTA was set at $14.0^{\circ}$.

Figure 8A shows the depth (axial position) vs the means and SDs of the measured BAs at each depth. Specifically, using the first moments of the spectra obtained for local echo sizes, $1.6 \times 3.2 \mathrm{~mm}^{2}$ (high spatial resolution) and $3.2 \times 6.4 \mathrm{~mm}^{2}$ (low spatial resolution), and instantaneous MA frequencies with a size of $0.5 \times 0.6 \mathrm{~mm}^{2}$ (the highest spatial resolution), almost the same means for the BAs are obtained: about $11.9^{\circ}$. In contrast with the axial displacement/strain measurements shown in the previous section, the order of the SDs is as follows: first moments of local spectra with a high spatial resolution $\left(1.6 \times 3.2 \mathrm{~mm}^{2}\right)$ $<$ low spatial resolution $\left(3.2 \times 6.4 \mathrm{~mm}^{2}\right)<$ instantaneous frequencies.

For the 1D AM, SFDM, and SFDM1 approaches, Figure $8 \mathrm{~B}$ shows the lateral displacements and lateral strains measured with or without BA data obtained using the first moments, whereas Figure 8C shows the corresponding statistics evaluated for a central circular region of the stiff inclusion using diameters of 5.0 and $6.4 \mathrm{~mm}$ for displacement and strain evaluations, respectively.

In Figure 8B, the lateral displacement images are shown using the original coordinate system: for methods using global echo rotation ( $\mathrm{r}: \alpha=11.9^{\circ}$ in Figure 3 for the conventional 1D AMr and SFDM1r), the measured lateral displacements are relocated by spatially rotating back with a BA of $11.9^{\circ}$. For the relocation calculation, a linear approximation was used. Nonrelocated displacements are omitted, because the linear approximation did not cause any significant errors 

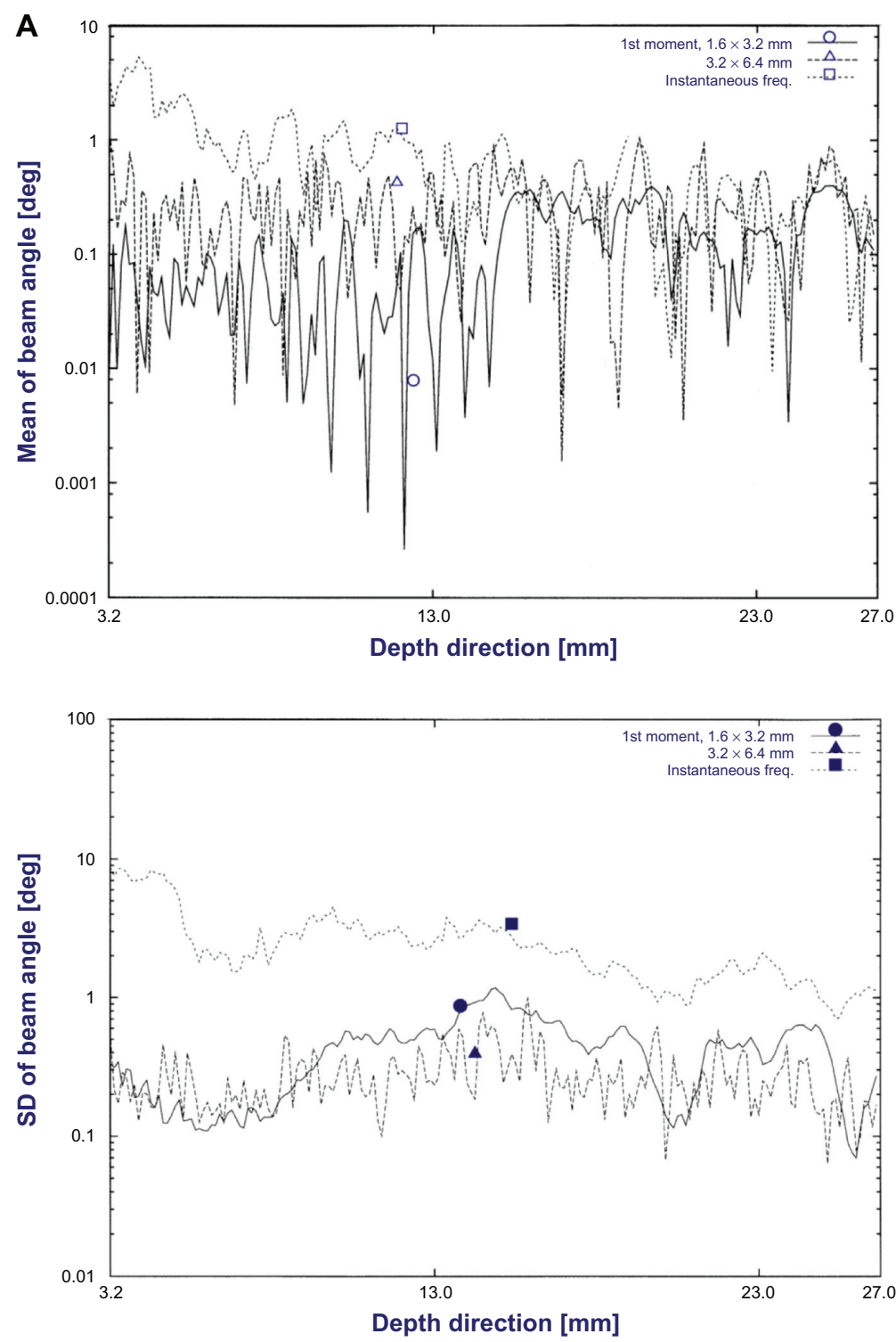

B

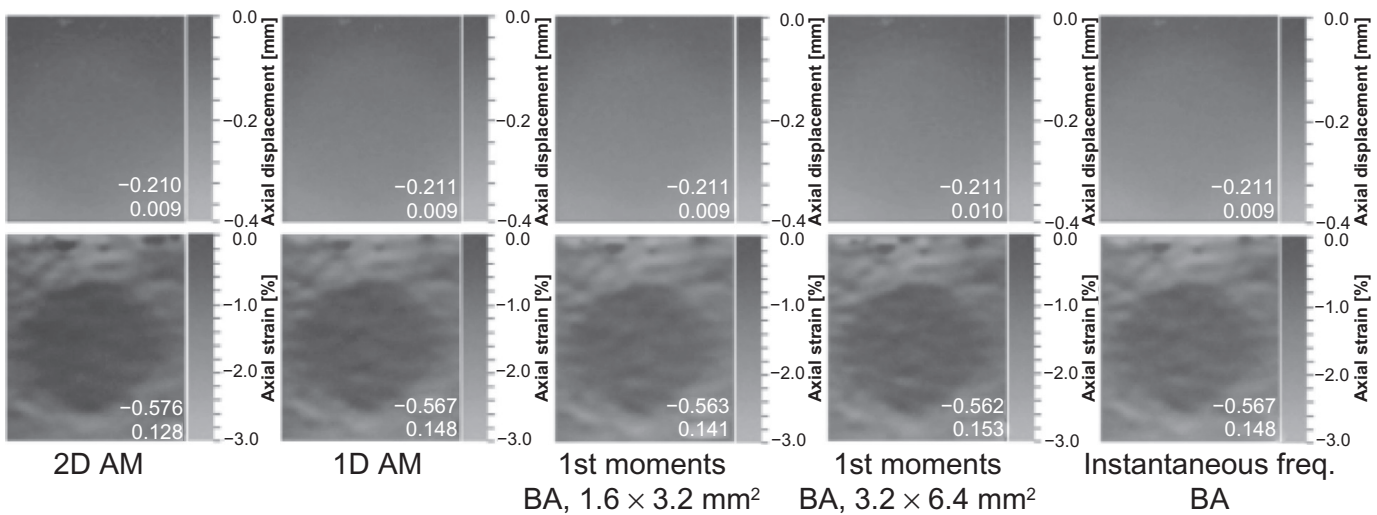

Figure 7 For a large global axial strain (ie, 1.5\%) for the same agar phantom as that in Figure 6, (A) the depth vs means and standard deviations (SDs) of the beam angles (BAs) are shown. Depth vs means and SDs were measured using the first moments of spectra obtained with a high spatial resolution $\left(\mathrm{I} .6 \times 3.2 \mathrm{~mm}{ }^{2}\right)$ and a low spatial resolution $\left(3.2 \times 6.4 \mathrm{~mm}^{2}\right)$ and using the instantaneous frequency moving average with $0.5 \times 0.6 \mathrm{~mm}^{2}$, respectively. (B) Grayscale images (region of interest: 23.8 [axial] $\times 19.4$ [lateral] at a depth of $3.2 \mathrm{~mm}$ ) of axial displacements and axial strains measured using the original two-dimensional autocorrelation method (2D AM) version (a type of spectra frequency division method), one-dimensional (ID) AM with no BA correction, and the ID AM using BAs with the corresponding three BA corrections. The means and SDs evaluated in the central circular region of the stiff inclusion are shown (for displacements, diameter $=5.0 \mathrm{~mm}$; strains, $6.4 \mathrm{~mm}$ ).

Abbreviations: deg, degrees; freq, frequency. 
(see Figure 8C; the means and SDs for the conventional 1D AMr are 0.8457 and $0.0230 \mathrm{~mm}$ vs 0.8456 and 0.0230 $\mathrm{mm}$; for SFDM1r, they are 0.8438 and $0.0229 \mathrm{~mm}$ vs 0.8438 and $0.0229 \mathrm{~mm}$ ).

All the lateral strains in Figure 8B, except for the conventional 1D AMr and SFDM1r method with nonrelocation, are also shown on the original coordinate system, obtained by applying a 2D differential filter with a cutoff frequency of $1.022 \mathrm{~mm}^{-1}$ (ie, spatially isotropically circular, as shown by equation 69 in Sumi et $\mathrm{al}^{36}$ ) to the lateral displacements expressed on the original coordinate system in the direction of the dominant deformation direction $\left(7.6^{\circ}\right)$, which is the most accurately measured angle data using the 2D AM and $\mathrm{LM}^{23}$ with respect to the lateral coordinate axis. For the conventional 1D AMr and SFDM1r, the nonrelocated lateral strains are also shown, and were obtained by applying the same 2D differential filter to the nonrelocated, measured lateral displacements in the dominant deformation direction of $-4.3^{\circ}\left(7.6^{\circ}-11.9^{\circ}\right)$.

In contrast, both the BA and BAr methods (ie, the BA approach) do not require any relocation of measurements. For all the nonrelocation measurements, including with the 1D AMr and the SFDM1r, the differential filter does not amplify the approximation errors included by the relocation of the displacements (ie, the measured strains should be relocated, not shown). However, the approximation errors that occur during echo rotation will be included in all of the measurements except for the BA method, so the BA method is not affected by any such errors. Measurement accuracy will be examined in detail later in this report.

As shown in Figure 8C, for the lateral displacement measurements using the 1D AM, SFDM, and SFDM1 approaches, the order of the SDs is as follows: BA or BAr (local rotation) methods $>$ methods using global echo rotation (r) with

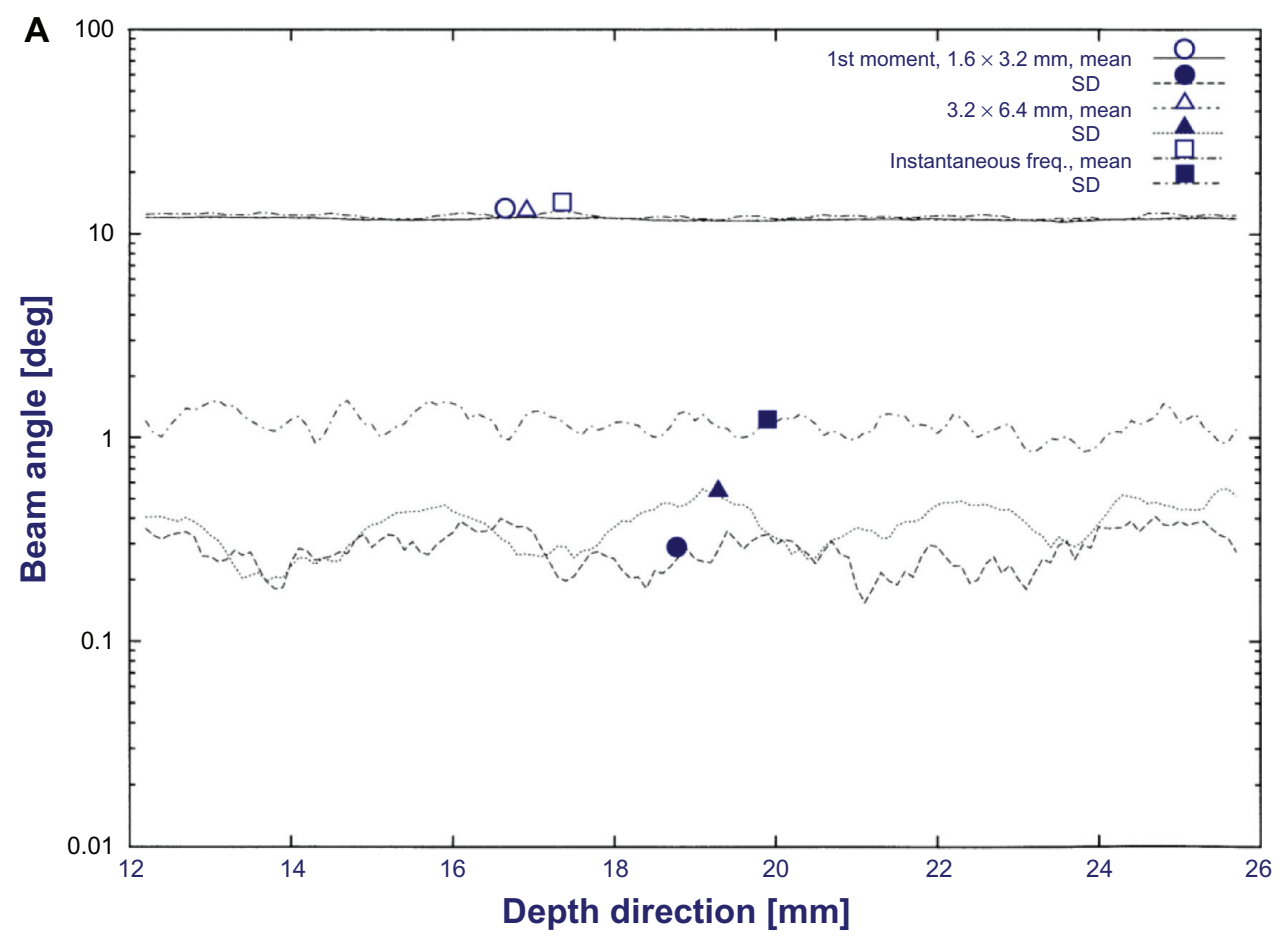

Figure 8 For an agar phantom compressed laterally, (A) shows the depth vs means and standard deviations (SDs) of the beam angles (BAs) measured using the first moments of spectra obtained with a high spatial resolution $\left(I .6 \times 3.2 \mathrm{~mm}^{2}\right)$ and a low spatial resolution $\left(3.2 \times 6.4 \mathrm{~mm}^{2}\right)$ and using the moving-averaged instantaneous frequencies with $0.5 \times 0.6 \mathrm{~mm}^{2}$, respectively. (B) Grayscale images of lateral displacements and lateral strains measured using the conventional one-dimensional autocorrelation method (AM) plus a global echo rotation $r\left(I D A M r ; r, 11.9^{\circ}\right), B A, B A$ plus local rotation (BAr), the spectra frequency division method (SFDM), the SFDM plus BA method (SFDMBA), the SFDM version I (SFDMIr; global rotation [r], II.9 ${ }^{\circ}$ ), and the SFDMIr plus BAr method (SFDMIBAr, local rotation), in addition to SFDM version 2 (SFDM2) and the lateral Doppler technique (AM) with global rotation $\left(r^{\prime}, 7.6^{\circ}\right)$ for a steering angel method (ASTA). Nonrelocated images are shown only for lateral strain images (ie, only obtained using global rotation [r, II.9 $9^{\circ}$ or $r^{\prime}, 7.6^{\circ}$ ] with the conventional ID AMr, SFDMIr, SFDM2, and the lateral AM). (C) The corresponding evaluated statistics (ie, the means and SDs) of lateral displacements and lateral strains in the central circular region in the stiff inclusion (diameter $=5.0$ and $6.4 \mathrm{~mm}$ for lateral displacements and lateral strains, respectively). The nonrelocated results are also shown for both the lateral displacements and the lateral strains. In the statistical results for the displacements, the magnitudes of the displacement vectors measured using two-dimensional (2D) AM and ID AM with demodulation for lateral modulation (LM), and the conventional ID AMr (global rotation [r], $\pm 11.9^{\circ}$ ), BA, BAr, the SFDM, the SFDMBA, the SFDMIr (global rotation [r], \pm II. $9^{\circ}$ ), and the SFDMIr plus BAr method (SFDMIBAr) for multiple crossed beams are also shown. The statistics for the angles of the displacement vectors are shown in Figure $9 \mathrm{C}$, together with other vector measurement results (ie, shear moduli and strain tensor components). The corresponding images are shown in Figure 9A (displacement vector components) and B (shear moduli and strain tensors). Abbreviations: conv., conventional; deg, degrees; Lat Dop, lateral Doppler technique; MCB, multiple crossed beam; SFDM2, spectra frequency division method version 2. 
B

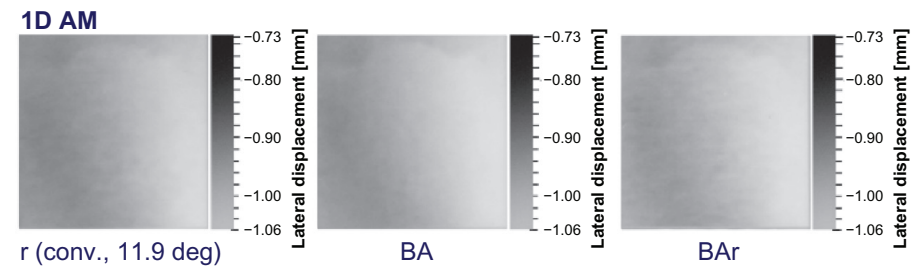

SFDM
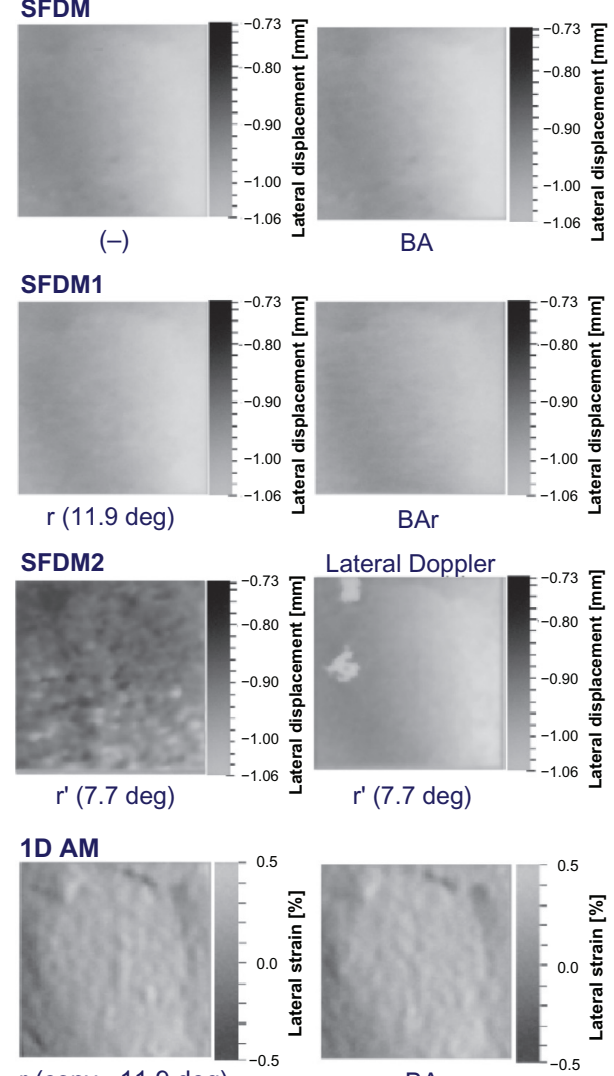

$r$ (conv., 11.9 deg)

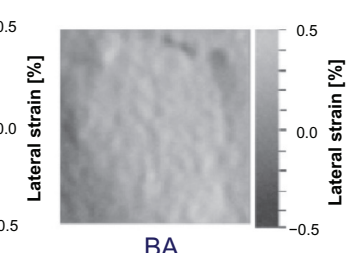

BA
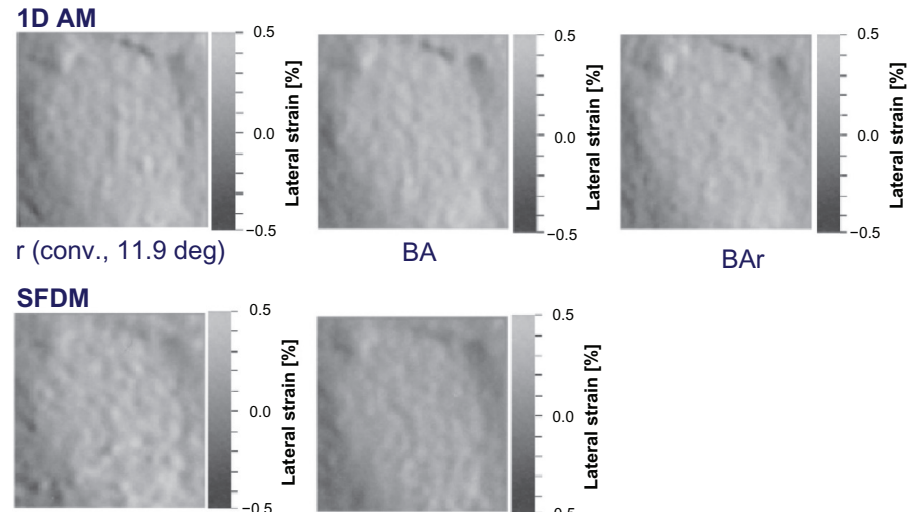

$(-)$

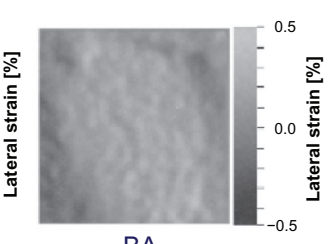

BA

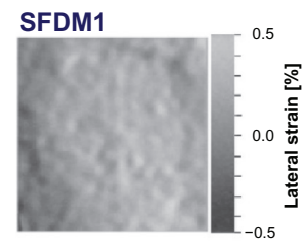

r (11.9 deg)

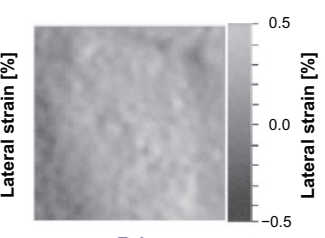

BAr

BAr

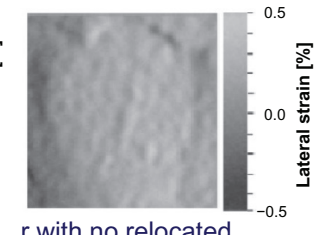

$r$ with no relocated

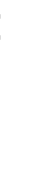

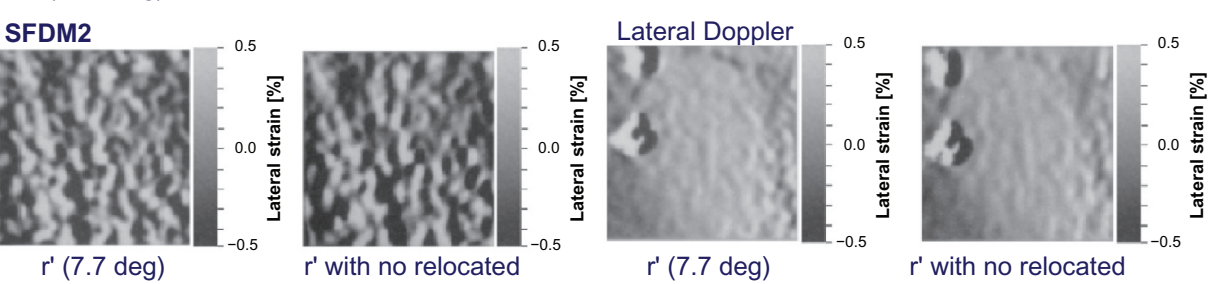

Figure 8 (Continued) 
Sumi et al

Dovepress
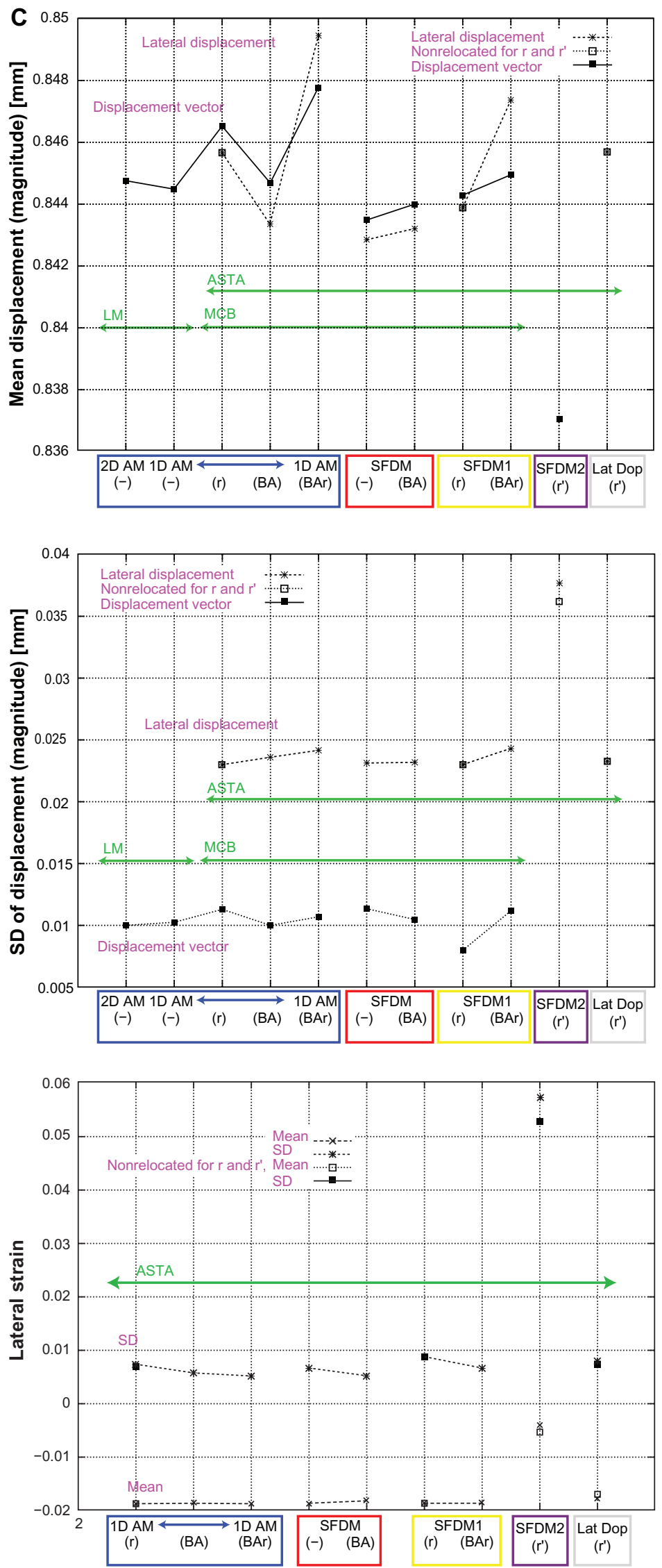

Figure 8

36

submit your manuscript | www.dovepress.com

Reports in Medical Imaging 2012:5 
relocation or nonrelocation. The SDs evaluated in the lateral displacement measurements exhibit neither rigid lateral motion nor measurement errors, even though the SDs are evaluated in the stiff inclusion. However, the magnitude of the lateral strains in the inclusion is rather uniform. For instance, data for displacements and strains generated in such a circular stiff region included in finite and infinite surrounding regions compressed uniformly in one direction can be found in Sumi ${ }^{20}$ and Sumi et $\mathrm{al}^{36}$ - these are numerical and analytic solutions, respectively. The lateral displacement measurements are affected by the generated axial displacement components. As shown in Figure 8C, compared with the statistics of the magnitudes of displacement vector measurements evaluated for the corresponding measurement methods used with MCB, the local displacement measurements are inaccurate: the means and SDs are small and large, respectively (eg, for the 1D AMBA, 0.8433 and $0.0236 \mathrm{~mm}$ vs 0.8447 and $0.0100 \mathrm{~mm}$ ). The measurement results obtained for such a displacement vector will be dealt with in detail shortly. The reason why the small means were obtained can be explained simply by the relationship between the directions of the beam and the target displacement (ie, inverse directions in the lateral direction). The order of measurement accuracy can be confirmed from the SDs of the lateral strains for which the means are almost the same: BA or BAr methods $<$ methods using global rotation (r) with nonrelocation $<$ methods using global rotation $(\mathrm{r})$ with relocation.

For the 1D AM approach, the SDs of the measured lateral strains (Figure 8C) are 0.0058 (BA), 0.0053 (BAr) $<0.0070$ ( $\mathrm{r}$ with nonrelocation) $<0.0072$ ( $\mathrm{r}$ with relocation). For the SFDM approach, the values are 0.0052 (BA) $<0.0067$ (original), and for the SFDM1r approach, the values are 0.0066 (BAr) $<0.0085$ ( $\mathrm{r}$ with nonrelocation) $<0.0087$ ( $\mathrm{r}$ with relocation). In this experiment, the $1 \mathrm{D} \mathrm{AM}$ and SFDM approaches yield almost the same measurement accuracy. However, this is different from the large lateral bandwidth case shown for axial displacement/strain measurements using a nonsteered beam, ${ }^{31}$ and for the echo data with a small lateral bandwidth obtained with beam steering; compare the corresponding spectra (first panel on the left) with that of the nonsteered case (the third panel from the left) shown in figure 4B in Sumi et al. ${ }^{23}$ The 1D AM and SFDM approaches yield a higher measurement accuracy than the SFDM1r approach, regardless of a BA (ie, with no rotation), BAr (ie, with local rotation), or global rotation (r) approach.

For comparison, the corresponding results obtained using the SFDM2 $2^{23}$ and the lateral Doppler technique ${ }^{17,18,23}$ are also shown in Figure 8A-C. These were obtained with a global echo rotation $r^{\prime}\left(7.6^{\circ}\right)$. Both the SFDM2 and the lateral Doppler technique yield inaccurate measurements. In the grayscale image for the SFDM2, the stiff inclusion cannot be detected, whereas in that of the lateral Doppler technique, clear measurement errors are detected. Inaccurate means and large SDs were confirmed for the corresponding lateral strain measurements $(-0.0054$ and $0.0526 ;-0.0172$ and 0.0073 vs 1D AMBA, -0.0180 and 0.0052$)$.

\section{Displacement vector/strain tensor measurements using $M C B$ versus $L M$}

The results of displacement vector measurements are described in this section. First, the same steered-beam echo data used for the lateral displacement measurements with ASTA shown in the previous section and the laterally, symmetrically steered-beam echo data (ie, the BAs are chosen and generated with values of $-14.0^{\circ}$ and $-11.9^{\circ}$, respectively) ${ }^{15,16,19,23}$ are used for comparisons of measurement accuracy with LM with the 2D AM vs MCB using BA or BAr methods (ie, 1D AMBA, 1D AMBAr, SFDMBA, and SFDM1BAr) and the corresponding global echo rotation methods (ie, 1D AMr, SFDM, and SFDM1r). For strain measurements, the 1D differential filter ${ }^{36}$ was used ${ }^{21,31}$ with almost the same cutoff frequency in both axial $\left(0.894 \mathrm{~mm}^{-1}\right.$; see equation 64 in Sumi et $\mathrm{al}^{36}$ ) and lateral directions as used with the 2D filter in the previous section.

Figure 9A and B show the grayscale images of measured displacement vector components, shear moduli, and 2D strain tensor components (the lateral, axial, and shear strains). In Figure $8 \mathrm{C}$, the statistics (the means and SDs) are evaluated for a central circular region of the stiff inclusion using diameters of 5.0 and $6.4 \mathrm{~mm}$ for the measured magnitudes of the displacement vectors in addition to those of the measured lateral displacements (as discussed in the previous section). Figure 9C shows those of other measurements: the angles of the displacement vectors, the strain tensor components, and the relative shear moduli obtained using the strain tensor components assuming a 2D stress condition (unity is used as a reference shear modulus value for relative shear modulus measurements). ${ }^{20,21,36}$

For comparison with LM, the grayscale images obtained with the 2D AM are shown in Figure 9A and B (the same images are also shown in figure 1A in Sumi and Shimizu ${ }^{19}$ ). The corresponding statistics are also shown in Figure 9C, in addition to those evaluated for measurements obtained with the 1D $\mathrm{AM}^{13,22}$ with demodulation (the corresponding grayscale images are shown in figure 1B in Sumi and Shimizu $\left.{ }^{19}\right){ }^{17-19}$ 
It is worth noting that in Figure 9B, the images of shear moduli are not shown for the methods using a conventional global echo rotation (r) - the 1D AMr and the SFDM1r. This is because dead regions for data occur at the four corners of the ROI because of the global echo rotation (see the four corners shown in the lateral strain images). In addition, a reference region for a shear modulus cannot be set at the right vertical borderlines for the 2D shear modulus measurements. By considering the characteristic curves of the partial differential equations where the coefficients are composed of the measured strain tensor components and the shear modulus is an unknown, a reference region should be selected so that the reference region extends in a direction that crosses the direction of a dominant deformation (in this experiment, the reference region and the dominant deformation in the axial and lateral directions, respectively). ${ }^{20,21,36,37}$

As expected, being different from the lateral displacement measurements, the ranking of the SDs for the displacement vector measurements (except for the SFDM1 approach), as well as for the strain tensor measurements is as follows: BA method (no rotation) $<$ BAr method (local rotation) $<$ global echo rotation (r). This exhibits the order of measurement accuracy (see those for displacements and strains in Figures 8C and 9C; those for displacement magnitudes and lateral strains for the 1D AM approach are $0.0100 \mathrm{~mm}$ and $0.00331<0.0107 \mathrm{~mm}$ and $0.00371<0.0112 \mathrm{~mm}$ and $0.00531 \mathrm{~mm})$. As shown by the strain measurements using the SFDM1 approach, SFDM1BAr (local echo rotation) is also more accurate than SFDM1r (global rotation [r]).

\section{A $\operatorname{LM~(\pm 11.9~deg)}$}

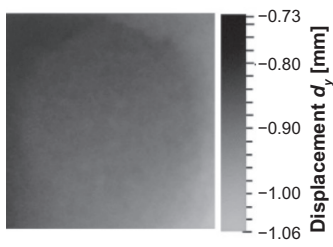

$2 \mathrm{D} \mathrm{AM}$

MCB ( $\pm 11.9 \mathrm{deg})$

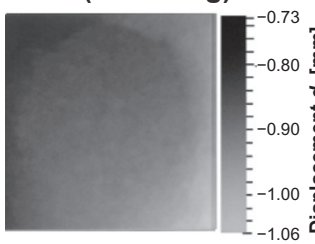

1D AM (BA)

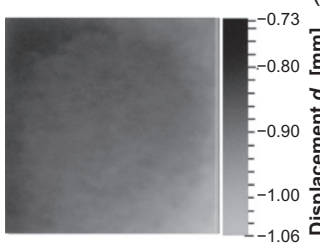

SFDM (-)

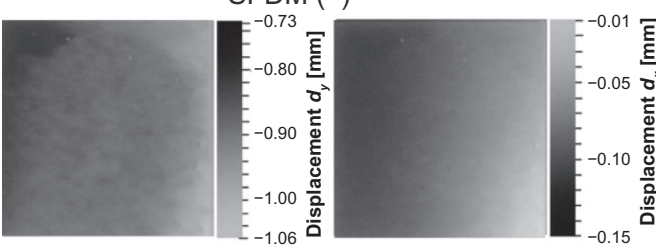

SFDM1 ( $r, \pm 11.9 \mathrm{deg})$

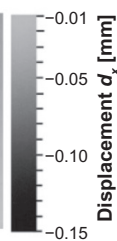

\section{MCB ( $\pm 11.9 \mathrm{deg})$}

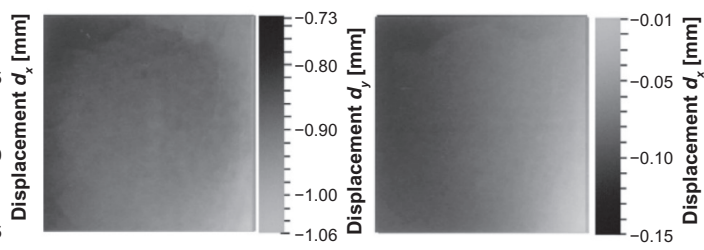

1D AM ( $r, \pm 11.9 \mathrm{deg})$

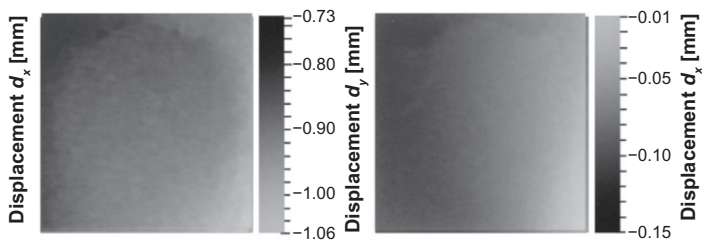

1D AM (BAr)

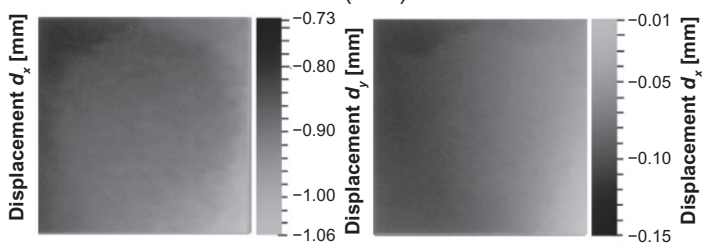

SFDM (BA)

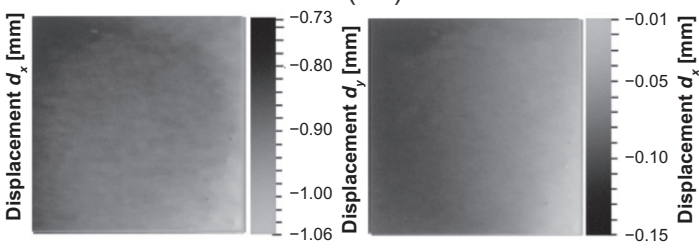

SFDM1 (BAr)

Figure 9 Grayscale images of $(\mathbf{A})$ displacement vector components and (B) shear moduli and strain tensor components measured using the two-dimensional autocorrelation method (2D AM) for developed lateral modulation (LM), and the one-dimensional (ID) AMr (ID AM plus a global echo rotation of $r$ ), the ID AM plus beam angle (BA) method (ID AMBA), the ID AMBA plus local rotation (ID AMBAr), the spectra frequency division method (SFDM), the SFDM plus BA method (SFDMBA), the SFDM version I (SFDMIr), and the SFDMIr plus BAr method (SFDMIBAr) for multiple crossed beams (MCBs). For the ID AMr and the SFDMIr, four dead regions (in the corners) are shown in lateral strains. (C) The corresponding evaluated statistics (ie, the means and standard deviations [SDs]) of the displacement vector angles, strain tensor components, and relative shear moduli in the central circular region of the stiff inclusion (diameter $=5.0$ and 6.40 mm for displacement vector components and for shear moduli and strain tensor components, respectively) and the statistics for ID AM with demodulation for LM (corresponding grayscale images are shown in figure IB in Sumi and Shimizu ${ }^{19}$ ). See Figure $8 \mathrm{C}$ for those of the corresponding displacement vector magnitudes.

Abbreviation: deg, degrees. 
B 2D AM with LM

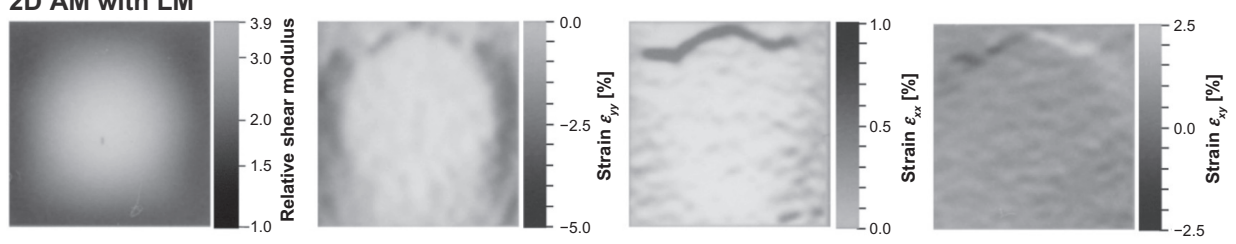

1D AMr with MCB

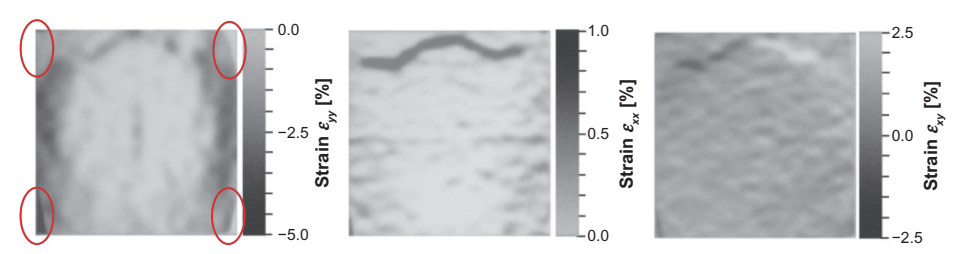

1D AMBA with MCB
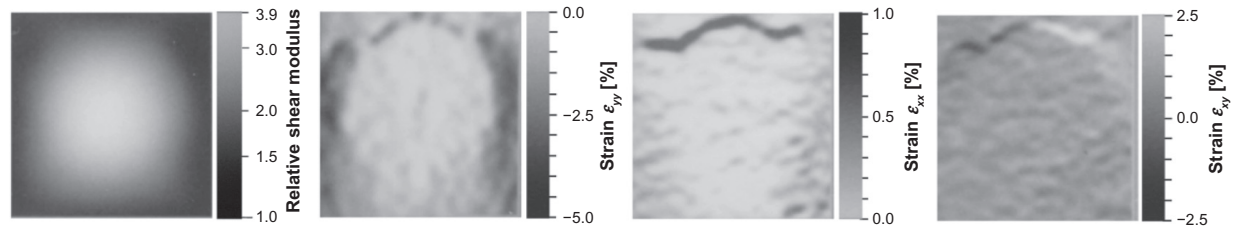

1D AMBAr with MCB
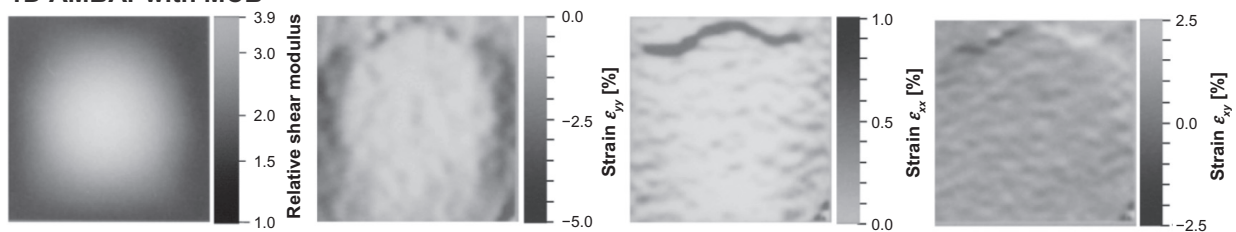

SFDM with MCB
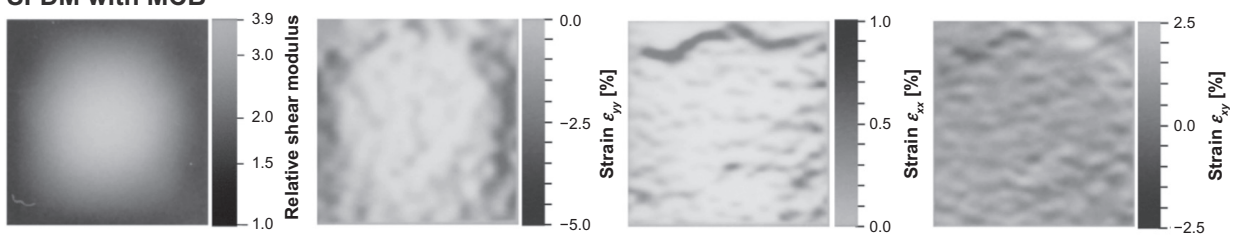

SFDMBA with MCB
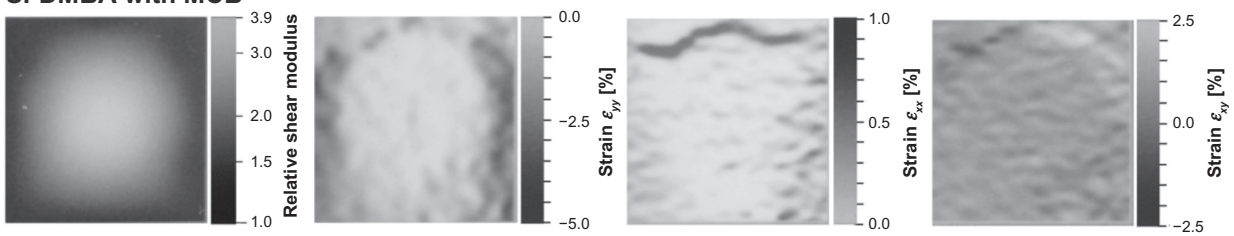

SFDM1r with MCB
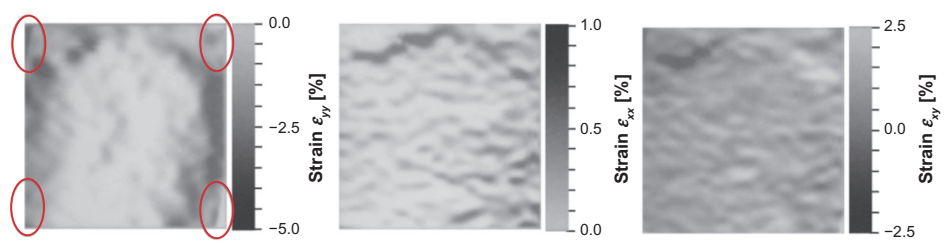

SFDMBA1r with MCB
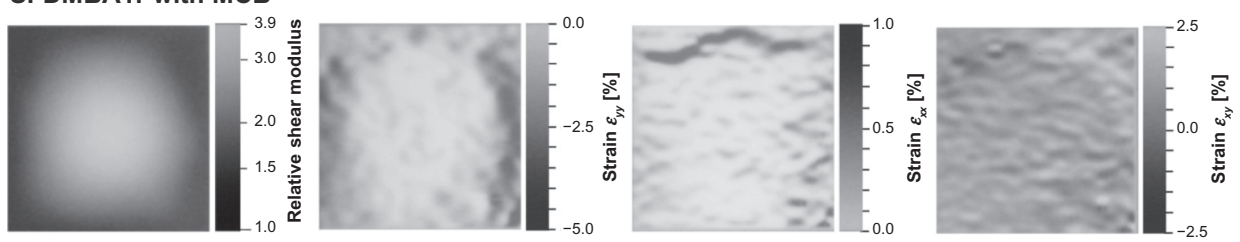

Figure 9 (Continued) 

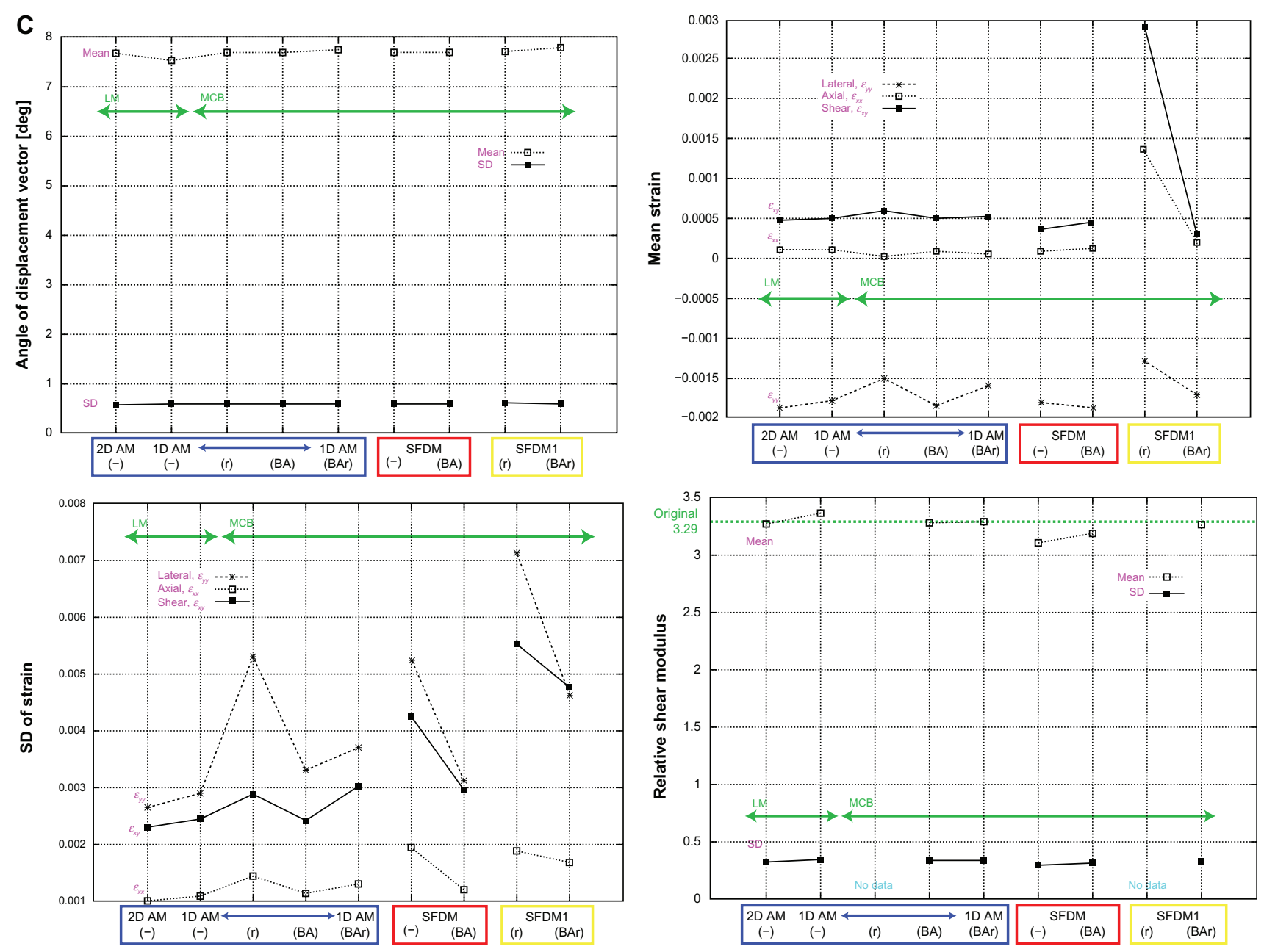

Figure 9

Similarly for the lateral displacement measurements, the 1D AM and SFDM approaches yield almost the same higher measurement accuracies than SFDM1, regardless of the method (ie, BA [no rotation], BAr [local echo rotation], or global echo rotation $[r])$. For accurate measurement methods using the BA approach, the relative shear modulus is also accurately evaluated (see the means [3.29] of the BA or BAr methods in Figure 9C). It is also worth noting that BA or BAr methods yield almost the same measurement accuracies as the $1 \mathrm{D} \mathrm{AM}^{13,22}$ with LM and demodulation ${ }^{17-19}$ (see the SDs of the displacement magnitudes and lateral strains, 0.0102 and 0.00290 , for instance), which does not exceed the accuracy achieved with the 2D AM with LM (the SDs of displacement magnitudes and lateral strains, are 0.0100 and 0.00267 , for instance). As mentioned in the explanation of multidimensional phase matching, the number of calculations is also larger than for the 2D AM with LM, particularly when using BAr methods. The quantitatively evaluated measurement accuracies can also be confirmed

visually in the images, particularly in the strain images (Figure 9B).

\section{Discussion and conclusion}

In real-world applications, the beam direction generated differs from the designed direction because of the directivities of transmission and reception apertures. Moreover, the direction of scattering, reflection, or an attenuation of a US signal, can change the direction of a US beam in different positions and produce frequency modulation. ${ }^{34}$ In this report, in order to increase the accuracy of a measurement of an arbitrary directional displacement such as an axial displacement using a nonsteered beam (eg, Sumi and Ebisawa ${ }^{31}$ ), a lateral displacement using ASTA (ie, a defined steering angle in Sumi et a ${ }^{17}$ and $\mathrm{Sumi}^{18}$ ), a displacement vector using $\mathrm{MCB}$ (ie, MCBs in Sumi ${ }^{13,18,20}$ and Sumi et $\mathrm{al}^{17}$ ), and all other displacement measurements using other beamforming methods (eg, a radial displacement not shown in this report), the spatial resolution in a generated BA is used in a novel manner: it is obtained by 
calculating the arctangent of the ratio of the axial and lateral instantaneous frequencies or the first moments of local spectra for $2 \mathrm{D}$ cases (Equation 1; see Appendix 2 for a $3 \mathrm{D}$ case). ${ }^{34}$ For ASTA and MCB measurements, generally, a rotation processing is performed globally on an echo data frame, an ROI, or a coordinate system (ie, global echo rotation) using the negative of the steering angles set during beamforming. To increase measurement accuracy with conventional global echo rotation, Sumi et $\mathrm{al}^{23}$ have used the first moments of the global spectra for the calculation of the BA. During practical signal rotation processing, however, artifacts that occur, such as sidelobes, must be removed by filters (this was not mentioned in the Lateral displacement/strain measurements using ASTA and Displacement vector/strain tensor measurements using MCB versus LM sections). ${ }^{17}$

When using the 1D AM with the multidimensional $\mathrm{MA},{ }^{13,22}$ the local displacement in the beam direction is accurately measured by dividing the local instantaneous phase change by the instantaneous frequency calculated in the beam direction, and then an arbitrary directional displacement, including a general axial displacement, can be accurately measured. This is performed with or without rotation of local echo data (ie, a local echo rotation). This is referred to in the methods for the BA approach - specifically, 1D AMBA (1D $\mathrm{AM}+\mathrm{BA})$ and $1 \mathrm{D} \mathrm{AMBAr}(1 \mathrm{D} \mathrm{AM}+\mathrm{BA}+$ local rotation $) .{ }^{34}$ Use of a multidimensional MA permits such measurements. Also, for BAr methods, the artifacts caused by an echo rotation processing are removed by filtering.

Also, it has been theoretically shown that the 1D AM with MCB but no echo rotation (ie, 1D AMBA with MCB) $)^{34}$ is equivalent to the $\mathrm{MAM}^{11-13}$ with $\mathrm{LM}^{1{ }^{1-19}}$ Using the $1 \mathrm{D}$ AMBA with ASTA, a more accurate lateral displacement measurement can be achieved than with the lateral Doppler technique. ${ }^{17,18,23,34}$ For comparisons with the 1D AMBA and the 1D AMBAr, the SFDM used in the MAM was also used, instead of the 1D AM (ie, the SFDMBA and the SFDM1BAr are obtained). ${ }^{34}$ Previously, for nonsteered beamforming, the original SFDM method yielded more accurate axial displacement measurements than the $1 \mathrm{D} \mathrm{AM} .^{31}$ For the evaluation of measurement accuracy achieved by considering the generated beams together with such methods, agar phantom experiments were performed, in which axial and lateral compressions were performed with no steering and steering (ASTA or $\mathrm{MCB})$, respectively.

In the experiments using ASTA or MCB, the effect of the SFDM was not confirmed because a small lateral bandwidth was generated (with almost the same accuracy as the methods in the 1D AM approach). For ASTA and MCB, the effectiveness of SFDM will be reported elsewhere using large lateral bandwidth echo data. However, in all the experiments, the new BA methods based on the 1D AM and SFDM approaches with no echo rotation processing yielded more accurate measurements with significantly fewer calculations than the corresponding methods using local echo rotation processing (ie, BAr methods), and those using conventional global rotation. Differing from the methods using a global or local echo rotation, the BA methods do not yield dead region data in an ROI, and they do not yield any measurement errors because of an approximate interpolation of echo data or of measured motion/deformation data. The approximate errors became slightly larger when using a small region of echo data with $3.2 \times 6.4 \mathrm{~mm}^{2}$ vs $12.8 \times 25.6 \mathrm{~mm}^{2}$ for the local rotation: for instance, SDs of 1D AMBA, of 2.41 vs $2.40 \times 10^{-2} \mathrm{~mm}$; those of the 1D SFDM1BAr were 2.49 vs $2.47 \times 10^{-2} \mathrm{~mm}^{2}$. However, the differences in the SDs led to large errors for the lateral strain measurements. In particular, the local echo rotations over the ROI made it difficult to accomplish the measurements in real time, even if such a small region was used. Although a perfect interpolation with no approximate errors can also be performed using a phase rotation of the target distribution in a Fourier domain, ${ }^{9,10,13}$ the interpolation is not practical in the sense that a real-time measurement is not possible.

Specifically, although measurements of arbitrary 1D displacements obtained with the BA methods (ie, axial with the axial compression and nonsteering; lateral with the lateral compression with ASTA) achieved higher accuracies than other one-directional displacement measurement methods (eg, the conventional and Doppler techniques, ${ }^{17,18,23}$ and so forth), the measurement accuracies achieved in the 1D displacement measurements are significantly lower than those of a displacement vector because of the practical 3D deformation or motion (eg, for lateral compression and $\mathrm{MCB}$, different accuracies in the means and about a half $\mathrm{SD}$ of the lateral displacement measurement). In terms of the amount of processing required, the MAM with LM is more efficient than methods with $\mathrm{MCB}$, as mentioned in the explanation of multidimensional phase matching. In the agar phantom experiments, the means and SDs of the displacement magnitudes in the $1 \mathrm{D}$ displacement measurements are respectively smaller and larger than those of the displacement vector measurements. If the directions of the crossed beam and displacement are the same, the means estimates will be inversely larger.

The one-directional BA methods with ASTA beamforming provide a simpler real-time manual technique for 
obtaining measurements for medical doctors or clinical staff than do the other one-directional measurement methods. LM beamforming permits a much simpler real-time manual technique without the use of an additional device required for the 1D measurements (such as an automatic detector for the direction of blood running through vessels). However, a larger number of beams are required to be generated, and for $2 \mathrm{D}$ and $3 \mathrm{D}$ displacement vector measurements, two and three crossed beams, respectively.

LM beamforming has also already enabled echo imaging (ie, B-mode imaging) with almost the same lateral resolution as the axial resolution, ${ }^{15,16}$ when the shapes of the apodization functions are carefully considered together with proper focusing methods. As used in the agar phantom experiments, the shapes should have large full widths at half maximum and short feet such as power functions rather than Hanning windows or Gaussian functions. For the transmissions, the crossed spherical focusing beams used in these experiments are better for obtaining a high measurement accuracy and a high-resolution echo image than crossed, laterally wide waves such as plane waves with no focusing. ${ }^{15,16}$

However, the transmission of such laterally wide waves is useful for the measurement and imaging of a rapid target motion such as blood flow in a carotid artery, because a more rapid scanning over an ROI can be achieved than with the transmission of conventional spherical focusing beams. ${ }^{13,15,16,23}$ Measurement results obtained on the same agar phantom by the combination of rapid US transmissions with the new BA methods (ie, with a steered plane wave or crossed, steered plane waves) are shown in Appendix 4, together with those obtained using BAr methods, and the 2D AM and the 1D AM with demodulation for LM. All of the steered-beam reception results were obtained using dynamic spherical focusing. For US transmission, a nonsteered plane wave was also used. ${ }^{13,15,16,23}$ The generated steering angles are smaller than those of crossed, steered, spherical focusing beams. Accordingly, the evaluated SDs of the measured BAs are also smaller. The order of measurement accuracies for the US transmissions was the same as the results obtained with the use of the 2D AM, shown in Sumi et $\mathrm{al}^{15}$ and Sumi and Tanuma: ${ }^{16}$ crossed, steered spherical focusing beams $>$ crossed, steered plane waves $>$ nonsteered plane waves. With regard to displacement vector and lateral displacement measurements, the same orders of measurement accuracy were obtained.

Alternatively, for LM and ASTA, the effect of a manual, mechanical, or electric steering angle, and a coordinate rotation angle on measurement accuracy was examined in observing the direction of tissue motion (in simulations and agar phantom experiments). ${ }^{23}$ For both $\mathrm{LM}^{13,15,16}$ and ASTA, ${ }^{17,18}$ the steering and rotation angles can control the frequencies of all axes. For LM, the combination of nonsteered and steered beams can also be used for a displacement vector measurement together with a conventional non-LM B-mode imaging. However, it was confirmed that for the MAM with LM and the SFDM with ASTA, echo or coordinate rotations that produce laterally symmetric conditions increased the measurement accuracy of displacement vectors (in simulations). ${ }^{23}$ For the SFDM with ASTA, according to the agar phantom experiment results mentioned, ${ }^{31}$ such results can be obtained if the lateral bandwidth is large. For comparison with the results obtained in this report, in Appendix 4, only results obtained using a nonsteered spherical focusing beam with the same parabolic apodization and a nonsteered plane wave with no apodization for respective transmissions, together with a nonsteered spherical focusing beam with a parabolic apodization for reception are shown. The use of a plane wave for transmission yielded inaccurate measurements. Interestingly, the transmission of the nonsteered spherical focusing beam yielded more accurate measurements than LM with a nonsteered plane wave transmission (Appendix 4). By using a proper apodization (ie, a rectangular apodization) with only a nonsteered spherical focusing beam, almost the same accuracy was obtained as with the most accurate original version of $\mathrm{LM}^{13,15,16}$ using crossed plural beams (not shown in this report). ${ }^{33,38}$ Disregarding lateral low frequency spectra $^{17,18,33,38}$ after completing beamforming (ie, summation of echo data with apodization and focusing) was particularly effective in such a case. In a manner similar to LM using crossed plural beams, also when using SFDM, lateral cosine and sine modulations are reversible after beamforming by changing the signs of the divided spectra. ${ }^{33,38}$ The respective lateral cosine and sine modulations are effective for yielding LM imaging and displacement vector measurements, respectively, from a single beam transmission. ${ }^{33,38}$ Details will be reported elsewhere.

For comparison of the new BA methods with ASTA or $\mathrm{MCB}$, and the 2D AM with LM, the $1 \mathrm{D} \mathrm{AM}{ }^{13,22}$ with LM and demodulation ${ }^{17-19}$ was also used. As discussed in Sumi and Shimizu, ${ }^{19}$ the measurement accuracy is lower than that of the 2D AM with LM. Even if decorrelation inherent with the use of the 1D AM is decreased by the use of the multidimensional $\mathrm{MA}^{13,22}$ and the multidimensional phase matching, ${ }^{9,10,13}$ if the asymmetry and differences in the beams passing through different paths (ie, the effects of different propagation speeds, attenuation, and scattering because of acoustic inhomogeneity, and frequency modulations, and so forth) are large, 
measurement accuracy decreases. In the present experiments even using the almost homogeneous agar phantom, inaccurate results were obtained. Echo rotation processing using the new BA calculation may also be effective for yielding symmetric beams in such a case, in addition to the case where symmetric beams cannot be obtained physically because of the existence of obstacles such as bone (however, the use of the 2D AM with LM requires less processing than the use of the digital demodulation method). ${ }^{17-19}$ In addition to the measurement accuracy shown, several advantages of ASTA beamforming (ie, using a single beam) over LM beamforming should also be reconfirmed (see Sumi et $\mathrm{al}^{17}$ or Sumi, ${ }^{18}$ or appendix A in Sumi et $\mathrm{al}^{23}$ ).

The SFDM approach has the potential for yielding higher accuracy for arbitrary 1D displacements than the 1D AM approach, and its combination with LM will also yield a higher accuracy for displacement vectors than the MAM with LM based on the 1D AMBA method. In addition to the use of a large lateral bandwidth generated using a rectangular apodization, ${ }^{33,38}$ a proper window for the spectra division must be sought for or designed. The spectra division will not always be performed symmetrically according to the independency of simultaneous equations, and echo SNRs and bandwidths to be obtained. Properly, partial spectra may be disregarded. Using the SFDM, an overdetermined system (ie, using the least squares estimation or superposition) can also be constructed for all the displacement measurements (arbitrary directional displacements such as axial, lateral, and radial, and displacement vectors and so forth), and all beamforming methods (ie, nonsteered, steered with a variable BA, radial beamforming, ASTA, LM, and so forth). ${ }^{38}$ For instance, the proper combination of the SFDM with conventional nonsteered (eg, the use of spectra divided by vertical and/or horizontal divisions together with undivided original spectra) and LM beamforming (eg, horizontal divisions) yields a more accurate measurement of an axial displacement or a displacement vector in a trade-off with increasing the number of calculations. Although the nonfocused beam will also be used, the SFDM is particularly effective for a large bandwidth signal or large bandwidth local spectra with a high echo signal-to-noise ratio (SNR). For an accurate measurement, a lateral frequency should also be large. ${ }^{13}$ However, the use of an overly large steering angle also decreases the echo SNR and bandwidth. Grating lobes generated must also be removed. ${ }^{17}$ Almost the combination with low echo SNR and small bandwidth echo signals decreases the measurement accuracy. For LM, the use of a large number of crossed beams is also effective for obtaining an overdetermined system, ${ }^{38}$ although additional beamforming is required. Although the use of high SNR echo signals with different lateral frequencies increases the measurement accuracy, the use simultaneous of a nonsteered beam with a high SNR but a small bandwidth decreases the measurement accuracy inversely, particularly when the SFDM rather than the 1D AM is used (as aforementioned, properly generated beam should be used). ${ }^{38}$ The proper number of generated beams or spectra divisions for such beamforming will also be clarified. Moreover, the incoherent superposition of divided spectra is also effective for reducing echo speckles. ${ }^{38}$ Results using these efforts will be reported in detail elsewhere.

It is also necessary to mention the differential filter used for calculating strain tensor data and mechanical properties such as the shear modulus shown in this report. For 2D and 3D displacement data, a separable filter requires fewer calculations than a nonseparable filter. In this report, for the calculations of lateral strains from an arbitrary directional displacement, a circular band-limited 2D differential filter (nonseparable) was used. ${ }^{36}$ Alternatively, for the calculation of strain tensor components and shear moduli, a 1D differential filter with a cutoff frequency and a low-pass filter with almost the same cutoff frequency were used. ${ }^{36}$

The 3D versions of all the measurements/images can also be used. In this report, for examining a 1D displacement measurement method, the $1 \mathrm{D} \mathrm{AM}^{13,22}$ was primarily used. However, as mentioned in the discussion of the BA approach, Sumi et al's ${ }^{13,19,22,31}$ previously developed 1D DM can also be used. As reported in Sumi, ${ }^{13,18}$ Sumi and Tanuma, ${ }^{16}$ and Sumi and Shimizu, ${ }^{19}$ with a low echo SNR, the 1D DM and the MDM yield higher measurement accuracies than the 1D AM and the MAM, respectively, and vice versa. Measurement results will also be reported elsewhere for the 1D DM using a BA approach.

The development of versatile, accurate real-time US tissue displacement measurement methods will increase the practical applications of displacement/strain measurements of biological tissues (eg, blood flow, motion/deformation of the heart, blood vessels, liver, skin, muscle, and so forth) under normal (spontaneous) or artificial (static compression/stretch, vibration, acoustic radiation force, and so forth) motion. ${ }^{20,21}$ The methods can also be applied to microscopic examination and to the monitoring of thermal treatments such as highintensity focused ultrasound treatments. ${ }^{21,22}$

\section{Disclosure}

The authors report no conflicts of interest in this work. 


\section{References}

1. Satomura S. Study of the flow patterns in peripheral arteries by ultrasonics. J Acoust Soc Jpn. 1959;15:151-158.

2. Barker DW. Pulsed ultrasonic Doppler blood-flow sensing. IEEE Trans Sonics Ultrason. 1970;17(3):170-184.

3. Kasai C, Namekawa K, Koyano A, Omoto R. Real-time two-dimensional blood flow imaging using an autocorrelation technique. IEEE Trans Sonics Ultrason. 1985;32(3):458-464.

4. Foster SG, Embree PM, O'Brien WR. Flow velocity profile via timedomain correlation: error analysis and computer simulation. IEEE Trans Ultrason Ferroelectr Freq Control. 1990;37(3):164-175.

5. Wilson LS, Robinson DE. Ultrasonic measurement of small displacements and deformations of tissue. Ultrason Imaging. 1982;4(1):71-82.

6. Ophir J, Céspedes I, Ponnekanti H, Yazdi Y, Li X. Elastography: a quantitative method for imaging the elasticity of biological tissues. Ultrason Imaging. 1991;13(2):111-134.

7. Trahey GE, Allison JW, von Ramm OT. Angle independent ultrasonic detection of blood flow. IEEE Trans Biomed Eng. 1987;34(12): 965-967.

8. Yagi S, Nakayama K. Local displacement analysis of inhomogeneous soft tissue by spatial correlation of rf echo signals. In: Proceedings of the 1998 World Federation of Ultrasound in Medicine and Biology Meeting; October 17-21, 1988; Washington, DC; 113.

9. Sumi C, Suzuki A, Nakayama K. Phantom experiment on estimation of shear modulus distribution in soft tissue from ultrasonic measurement of displacement vector field. IEICE T Fund Electr. 1995;E78A(12):1655-1664.

10. Sumi C. Fine elasticity imaging on utilizing the iterative RF-echo phase matching method. IEEE Trans Ultrason Ferroelectr Freq Control. 1999;46(1):158-166.

11. Sumi C. Digital measurement method of tissue displacement vector from instantaneous phase of ultrasonic echo signal. Technical Report of Japan Society of Ultrasound Medicine; Dec 2002; Tokyo, Japan;pp. 37-40.

12. Sumi C. Multidimensional displacement vector measurement methods utilizing instantaneous phase [CD-ROM]. In: Proceedings of the 27th Annual International Conference of the IEEE Engineering in Medicine and Biology Society; September 1-4, 2005; Shanghai, China.

13. Sumi C. Displacement vector measurement using instantaneous ultrasound signal phase:multidimensional autocorrelation and Doppler methods. IEEE Trans Ultrason Ferroelectr Freq Control. 2008; 55(1):24-43.

14. Sumi C. Improvement of measurement accuracy of displacement vector by lateral modulation. In: Proceedings of the 2004 Autumn Meeting of the Acoustical Society of Japan; Sep 2004; Okinawa, Japan. pp. 1353-1354. Japanese.

15. Sumi C, Noro T, Tanuma A. Effective lateral modulations with applications to shear modulus reconstruction using displacement vector measurement. IEEE Trans Ultrason Ferroelectr Freq Control. 2008;55(12):2607-2625.

16. Sumi C, Tanuma A. Comparison of parabolic and Gaussian lateral cosine modulations in ultrasound imaging, displacement vector measurement, and elasticity measurement. Jpn J Appl Phys. 2008; 47(5B):4137-4144.

17. Sumi C, Matsui N, Shimizu K, Takanashi Y. Preliminary experiments on virtual source for lateral modulation. In: Proceeding of the 8th International Conference on the Ultrasonic Measurement and Imaging of Tissue Elasticity; September 14-17, 2009; Vlissingen, the Netherlands; http://www. elasticityconference.org/prior_conf/2009/PDF/088SumiC02FP.pdf.

18. Sumi C. Utilization of an ultrasonic beam steering angle for measurements of tissue displacement vector and lateral displacement. Rep Med Imaging. 2010;3:61-68.

19. Sumi C, Shimizu K. Ultrasonic agar phantom experiment for comparison of the measurement accuracy of tissue elasticity obtained by displacement vector measurement using lateral modulation with multidimensional autocorrelation and Doppler methods and corresponding one-dimensional methods. Rep Med Imaging. 2011;4:39-46.
20. Sumi C. Usefulness of ultrasonic strain measurement-based shear modulus reconstruction for diagnosis and thermal treatment. IEEE Trans Ultrason Ferroelectr Freq Control. 2005;52(10):1670-1689.

21. Sumi C. Relative shear modulus reconstruction for visualization with no geometrical artifact. Acoust Sci Technol. 2010;31(5):347-359.

22. Sumi C. Displacement measurement method and apparatus, strain measurement method and apparatus, elasticity and viscoelasticity constants measurement apparatus, and the elasticity and viscoelasticity constants measurement apparatus based treatment apparatus. Japanese patent 4260523. April 30, 2009 (application April 25, 2002); United States patent 7775980 B2 (application December 23, 2002).

23. Sumi C, Ichimaru K, Shinozuka Y. Beam steering and coordinate system rotation improves accuracy of ultrasonic measurements of tissue displacement vector and lateral displacement. Rep Med Imaging. 2011;4:47-66.

24. Jensen JA, Munk P. A new method for estimation of velocity vectors. IEEE Trans Ultrason Ferroelectr Freq Control. 1998;45(3):837-851.

25. Jensen JA. A new estimator for vector velocity estimation. IEEE Trans Ultrason Ferroelectr Freq Control. 2001;48(4):886-894.

26. Anderson ME. Multi-dimensional velocity estimation with ultrasound using spatial quadrature. IEEE Trans Ultrason Ferroelectr Freq Control. 1998;45(3):852-861.

27. Anderson ME. A heterodyning demodulation technique for spatial quadrature. In: Proceedings of the 2000 IEEE International Ultrasonics Symposium; October 22-25, 2000; San Juan, Puerto Rico; 1487-1490.

28. Fox MD. Multiple crossed-beam ultrasound Doppler velocimetry. IEEE Trans Sonics Ultrason. 1978;25(5):281-286.

29. Zahiri Azar R, Baghani A, Salcudean SE, Rohling R. 2D high-framerate dynamic elastography using delay compensated and angularly compounded motion vectors: preliminary results. IEEE Trans Ultrason Ferroelectr Freq Control. 2010;57(11):2421-2436.

30. Tanter M, Bercoff J, Sandrin L, Fink M. Ultrafast compound imaging for 2D motion vector estimation: application to transient elastography. IEEE Trans Ultrason Ferroelectr Freq Control. 2002;49(10):1363-1374.

31. Sumi C, Ebisawa T. Phantom experiments of axial strain measurements using multidimensional autocorrelation method, multidimensional Doppler method and direct strain measurement method. Acoust Sci Technol. 2009;30(2):117-123.

32. Sumi C. Ultrasonic measurement and imaging with lateral modulation: echo, tissue motion and elasticity. In: Tanabe M, editor. Ultrasound Imaging. Rijeka, Croatia: InTech; 2011:113-138.

33. Sumi C. Multidimensional ultrasonic imaging and displacement vector measurement using spectra frequency division method. IEICE Technical Report. 2011;US-07:85-90. Japanese.

34. Sumi C. Increase in accuracy of echo imaging and displacement measurement by considering the direction of beam-steering application to lateral modulation (LM) and a steering angle (ASTA). IEICE Technical Report. 2011;US-05:1-8. Japanese.

35. Sumi C. Ultrasonic axial strain measurement for lateral tissue deformation. Ultrasound Med Biol. 2007;33(11):1830-1837.

36. Sumi C, Suzuki A, Nakayama K. Estimation of shear modulus distribution in soft tissue from strain distribution. IEEE Trans Biomed Eng. 1995;42(2):193-202.

37. Sumi C, Nakayama K. A robust numerical solution to reconstruct a globally relative shear modulus distribution from strain measurements. IEEE Trans Med Imaging. 1998;17(3):419-428.

38. Sumi C, Takahashi Y, Ishii Y, Yamazaki N. A lateral modulation imaging using simple nonsteering beamforming. In: Abstract of the 10th International Conference on the Ultrasonic Measurement and Imaging of Tissue Elasticity; October 12-15, 2011; Arlington, TX, p. 73. Also in: Proceedings of The International Tissue Elasticity Conference. Available from: http://www.elasticityconference.org/prior_conf/2011/ PDF/064SumiC02FP.pdf. Accessed January 23, 2011. Abstract published October 12, 2011. 


\section{Appendices}

\section{Appendix I: Displacement measurements using beam steering and global echo rotation $(r)$ with inaccurate $B A$ data $^{34}$}

A conventional method using 1D measurements (ie, the 1D AM, ${ }^{3}$ Sumi et al's 1D AM, or the 1D DM method with a multidimensional $\mathrm{MA}^{13,19,22,31}$ ), beam steering, and global echo rotation (r) are reviewed (1D AMr and 1D DMr) to obtain the new displacement measurement methods referred to as BA (beam angle) and BAr (BA + local rotation) methods for an arbitrary directional displacement measurement. Generally, for a 2D ROI, the displacement in a direction expressed by inaccurate BA data $\alpha^{\prime}$ is measured using a nominal US frequency $f_{0}$, or the first moment or the instantaneous frequency $f_{0}^{\prime}$ in the beam (ie, in the axial) direction calculated after rotating an echo data frame or an ROI or a coordinate system (ie, a global echo rotation) by the angle $-\alpha^{\prime}$ (see $\alpha$ instead in Figure 3A and B). The inaccurate BA data $\alpha^{\prime}$ is a value used in performing analogue or digital beamforming to generate a designed beam or angle expressed by defined delays in the respective US elements (ie, not an accurate angle $\alpha$ expressed by Equation 1 and shown in Figure 3A and B). In the authors' case, ${ }^{23}$ for the global echo rotation, in practice, the global BA data calculated by Equation 1 using the first moments $\left(f_{x}^{\prime}, f_{y}^{\prime}\right)$ of global spectra have been used. However, the global BA data are still less accurate than the local BA data expressed by Equation 1 in the BA approach. In such a 2D case, a Fourier 2D transform (Figure 3A and B) or 1D transform (Figure 3C) is used, and the inaccurate displacement is obtained as

$$
d(x, y)=\Delta \theta(x, y) / f_{0} \text { or } d(x, y)=\Delta \theta(x, y) / f_{0}^{\prime} .
$$

Accordingly, the tissue displacement $d_{\beta}(x, y)$ with an angle $\beta$ (Figure 2) is calculated using inaccurate displacement data $d(x, y)$ and inaccurate BA data $\alpha^{\prime}$ as follows:

$$
d_{\beta}(x, y)=d(x, y) / \cos \left(\beta-\alpha^{\prime}\right) .
$$

Moreover, as mentioned in the explanation for the 1D AMBA, in a nonsteered case a BA $\alpha^{\prime}$ is often set to zero. In the 1D $\mathrm{AMr}$ case, the tissue displacement $d_{\beta}(x, y)$ is calculated by

$$
\begin{aligned}
d_{\beta}(x, y) & =d(x, y) / \cos \beta \\
& =\Delta \theta(x, y) / f_{x} \cos \beta .
\end{aligned}
$$

The calculation can be compared with the 1D AMBA (no echo rotation) or the 1D AMBAr (with local echo rotation) using Equations 1-3 or 3', whereas in the 1D DM approach, the calculation can be compared between the 1D DMr and the 1D DMBA or the 1D DMBAr. On the basis of the measurement of a generated BA, a measurement error is not caused by the fact that $f_{y} \sin \beta$ does not equal zero. If the lateral frequency fluctuation (ie, $f_{y}$ ) generated or the tissue displacement angle $\beta$ is large, the measurement error increases.

For a 3D case, using an inaccurate rotational angle $\varphi$ and polar angle $\theta$ to express the beam direction (see Figure A1), $3 \mathrm{D}$ echo data are rotated such that the beam direction corresponds to the axial direction, similar to the $2 \mathrm{D}$ case, and the displacement with an inaccurate direction is measured using a nominal US frequency $f_{0}$, or the first moment, or an instantaneous frequency $f_{0}^{\prime}$ in the axial direction. A 3D, 2D or 1D Fourier transform is performed.

\section{Appendix 2: Displacement measurements using a BA approach for a 3D ROI ${ }^{34}$}

As shown in Figure A1, when dealing with a 3D ROI, when the first moments or instantaneous frequencies are expressed as $\left(f_{x}, f_{y}, f_{z}\right)$, the beam direction at a point of interest $(x, y, z)$ can be expressed using a rotational angle $\varphi$ and a polar angle $\theta$ that are calculated as follows:

$$
\begin{gathered}
\tan \varphi=f_{y} / f_{x}, \\
\cos \theta=\mathrm{f}_{\mathrm{z}} / \sqrt{\left(f_{x}^{2}+f_{y}^{2}+f_{z}^{2}\right) .}
\end{gathered}
$$

Correspondingly, the displacement $d(x, y, z)$ in the beam direction can be accurately obtained by dividing the instantaneous phase change $\Delta \theta(x, y, z)$ between two frames calculated using a multidimensional $\mathrm{MA}^{11-13}$ by $\sqrt{\left(f_{x}^{2}+f_{y}^{2}+f_{z}^{2}\right)}$, as follows:

$$
d(x, y, z)=\Delta \theta(x, y, z) / \sqrt{\left(f_{x}^{2}+f_{y}^{2}+f_{z}^{2}\right)} .
$$

For instance, by detecting the direction of a tissue displacement $d_{\beta}(z, y, z)$ geometrically, such as from a corresponding US or other image, the target displacement $d_{\beta}(z, y, z)$ can be accurately measured in a manner similar to that for a $2 \mathrm{D}$ ROI case.

\section{Appendix 3: 3D displacement vector measurements using $M C B$ with a $B A$ approach $^{34}$}

For a 3D displacement vector measurement at a point of interest $(x, y, z)$ in a 3D ROI, for displacements in the respective directions, three crossed beams are measured using one of the new BA methods. For instance, when displacements 
$d_{1}(x, y, z), d_{2}(x, y, z)$ and $d_{3}(x, y, z)$ are measured in three beam directions which are expressed using rotational and polar angles, ie, $\left(\varphi_{1}, \theta_{1}\right),\left(\varphi_{2}, \theta_{2}\right)$ and $\left(\varphi_{3}, \theta_{3}\right)$, the tissue displacement vector $D=(d x, d y, d z)$ can be calculated by solving the simultaneous equations:

$$
\begin{aligned}
& \sin \theta_{1} \cos \varphi_{1} d x+\sin \theta_{1} \sin \varphi_{1} d y+\cos \theta_{1} d z=d_{1} \\
& \sin \theta_{2} \cos \varphi_{2} d x+\sin \theta_{2} \sin \varphi_{2} d y+\cos \theta_{2} d z=d_{2} \\
& \sin \theta_{3} \cos \varphi_{3} d x+\sin \theta_{3} \sin \varphi_{3} d y+\cos \theta_{3} d z=d_{3},
\end{aligned}
$$

where the rotational and polar angles are defined in a different manner from the steering angles $\alpha_{1}$ and $\alpha_{2}$ used in the 2D ROI case shown in Figure 5, and the positive directions of all the angles are set respectively in the same directions as defined in Figure A1.

Also in this $3 \mathrm{D}$ case, the equations derived geometrically using frequencies $\left(f_{x}, f_{y}, f_{z}\right)$ in three orthogonal directions (Figure A1) are equivalent to those obtained with LM.

\section{Appendix 4: Lateral displacement and displacement vector measurements in agar phantoms using plane waves and/or nonsteered beams for ASTA, MCB, and LM}

Using beamforming designs other than ASTA, MCB, and LM, and using the transmission and reception of steered spherical focusing beams with a dynamic focus (see the generated steering angles shown in Figure $8 \mathrm{~A}$, of about $\pm 11.9^{\circ}$ vs a designed $\pm 14.0^{\circ}$, and the measurement results shown in Figures $8 \mathrm{~B}$ and $\mathrm{C}$ and 9), the lateral displacement and displacement vector measurements performed on the

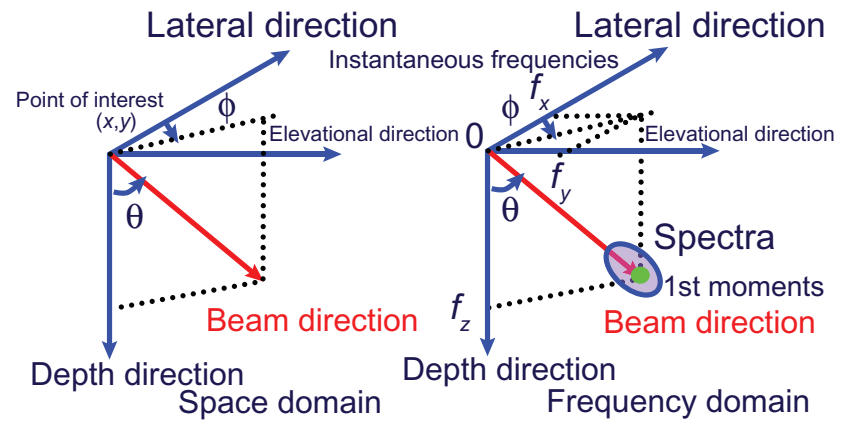

Figure AI In a three-dimensional case, schematic of beam direction generated and rotational angle $\varphi$ and polar angle $\theta$ and first moments of spectra or instantaneous frequencies $\left(f_{x}, f_{y}, f_{z}\right)$ at a point of interest $(x, y, z)$. same laterally compressed agar phantom are shown in this appendix. Here, only the 1D AM approach was used (ie, the SFDM approach was not used). The same differential filters are used for the calculations of lateral strains and strain tensors.

Figure $\mathrm{A} 2 \mathrm{~A}$ and $\mathrm{A} 2 \mathrm{~B}$ shows the means and SDs of measured BAs (Figure A2A), and grayscale images of the lateral displacements and lateral strains, measured using the global rotation (r), BA, and BAr methods (Figure A2B), for the transmission of a steered plane wave (ie, nonfocused) with the same steering angle as that of the received, steered spherical focusing beams with a dynamic focus and for the transmission of a nonsteered plane wave (ie, nonfocused with ASTA of $0^{\circ}$ ), respectively. For the strain measurements using a global rotation with accurate BA data (obtained using the first moments of global spectra), nonrelocated results are also shown (in this case, the strain data should be relocated, not shown). Moreover, Figure A2C shows (for MCB and LM using the same transmission and reception with the corresponding, laterally symmetric beams) the grayscale images of the displacement vector components, strain tensor components, and shear moduli (using a 2D stress assumption ${ }^{20,21,36}$ ) measured using the global rotation (r), BA, and BAr methods with $\mathrm{MCB}$, and the 2D $\mathrm{AM}^{11-13}$ and the $1 \mathrm{D} \mathrm{AM}{ }^{13,22,31}$ with demodulation ${ }^{17-19}$ with LM. The designed and generated steering angles are $\pm 14.0^{\circ} \mathrm{vs} \pm 11.9^{\circ}$ and $\pm 7.1^{\circ}$ vs $\pm 6.4^{\circ}$, respectively. Both of the evaluated SDs of the BAs are smaller than those of the dynamic focused transmission of steered spherical focusing beams (Figure A2A vs Figure 8A). However, such laterally wide wave transmissions are effective for completing a scan over an ROI in a target within a short time. ${ }^{13,15,16,23}$

At a glance, the measurements obtained with both of the plane wave transmissions are less stable than the corresponding measurements obtained with the dynamically focused transmission of steered spherical focusing beams (Figures $\mathrm{A} 2 \mathrm{~B}$ and $\mathrm{A} 2 \mathrm{C}$ vs Figures $8 \mathrm{~B}$ and $9 \mathrm{~A}$ and B), particularly those with transmission of a nonsteered plane wave rather than those with steered plane waves. The measurement accuracies were also evaluated and confirmed statistically, as shown in Figure A2D, together with those obtained for the transmission of steered spherical focusing beams (Figures $8 \mathrm{C}$ and 9C). Previously, the measurement accuracies were evaluated only for the 2D AM with LM. ${ }^{13,15,16}$ Also for the new BA or BAr methods with MCB, the same order of measurement accuracy was confirmed: dynamically focused transmission of steered spherical focusing beams $>$ transmission 
of steered plane waves $>$ transmission of a nonsteered plane wave. Also, as confirmed by Figure A2A-A2D, in a manner similar to that for dynamically focused transmission of steered spherical focusing beams (Figures 8 and 9), the order of measurement accuracy for both of the plane wave transmissions is $2 \mathrm{D} \mathrm{AM}>1 \mathrm{D} \mathrm{AM}$ with demodulation $>$ $\mathrm{BA}$ method (no rotation) $>$ BAr method (local rotation) $>\mathrm{r}$ method (global rotation). The measurement accuracy of the displacement vector is also higher than that using lateral displacement. For the global echo rotations (r), the four dead corners were also generated (thus, the relative shear modulus measurements were not obtained). All of these were obtained using the reception of steered dynamic spherical focusing beams.

In addition, in Figure A2D, the statistics were evaluated and they are also shown for the 2D AM and 1D AM with demodulation using no steered beam - that is, using the reception with nonsteered dynamic spherical focusing beams together with the transmission with nonsteered dynamic spherical focusing beams (see images in figure $1 \mathrm{E}$ and $\mathrm{F}$ in Sumi and Shimizu ${ }^{19}$ ), and the transmission with a nonsteered plane wave (images not shown). Both of the designed BAs are $0^{\circ}$; and the corresponding synthesized BA from the laterally symmetric spectra frequency division (ie, a synthesized LM using nonsteering, or the SFDM using a version of ASTA with nonsteering shown in figure $1 \mathrm{~B}$ with $\theta=0^{\circ}$ in Sumi et $\mathrm{al}^{23}$ ) have angles of $3.4^{\circ}$ and $2.6^{\circ}$, respectively. Although the synthesized BAs for the transmission and reception of nonsteered dynamic spherical focusing beams $\left(3.4^{\circ}\right)$ are smaller than those obtained by the transmission of the nonsteered plane wave with reception of the steered spherical focusing beams $\left( \pm 6.4^{\circ}\right)$, the corresponding images obtained are stable (figure $1 \mathrm{E}$ and $\mathrm{F}$ in Sumi and Shimizu ${ }^{19}$ ). Also, it is worth noting the more accurate means and the smaller SDs, except for the lateral and shear strains, than for the values obtained using a transmission of the nonsteered plane wave and a reception of the steered spherical focusing beams (Figure A2D). Moreover, for transmission, the use of a nonsteered, nonfocused plane wave yields less accurate and less stable measurements than the use of steered or nonsteered, dynamic spherical focusing beams (see Figure A2D). Such measurements using no steering and no focused beams will be improved with the $\mathrm{SFDM}^{23}$ and details will be reported elsewhere.
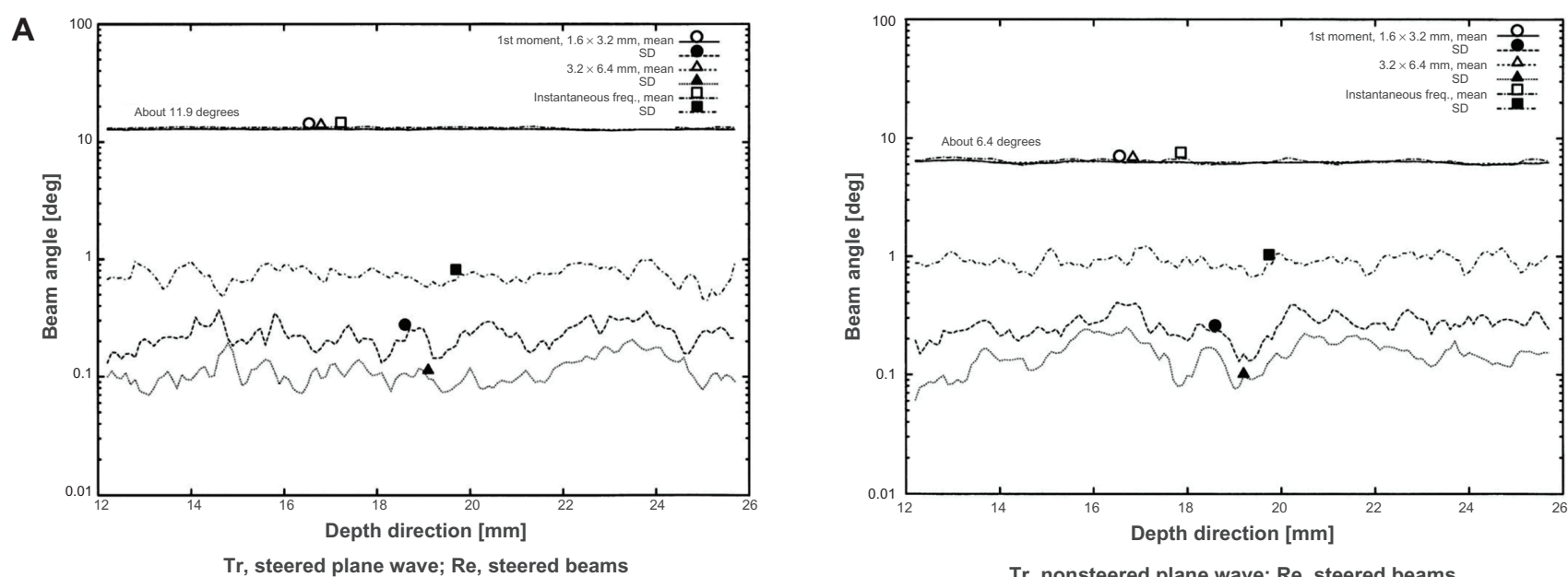

$\mathrm{Tr}$, nonsteered plane wave; Re, steered beams

Figure A2 For the respective transmissions of steered plane waves and a nonsteered plane wave, (A) depth vs means and standard deviations (SDs) of the beam angles (BAs) measured using the first moments of spectra obtained with a high spatial resolution $\left(1.6 \times 3.2 \mathrm{~mm}^{2}\right)$ and with a low spatial resolution $(3.2 \times 6.4 \mathrm{~mm})$ and using the instantaneous frequency moving average with $0.5 \times 0.6 \mathrm{~mm}^{2}$, respectively. Grayscale images of (B) lateral displacements and lateral strains and (C) displacement vector components, shear moduli, and strain tensor components measured using the two-dimensional autocorrelation method (2D AM) and the one-dimensional (ID) AM with demodulation for lateral modulation (LM), and the ID AM plus local rotation (AMr), the ID AM plus BA (ID AMBA) and the ID AMBA plus local rotation (ID AMBAr) for multiple crossed beams (MCBs). The four dead regions (corners) are shown for the ID AMr (ID AM plus a global echo rotation of $r$ ) with both transmissions. (D) The corresponding evaluated statistics (ie, the means and SDs) of the displacement vector magnitudes and angles, strain tensor components, and relative shear moduli in the central circular region in the stiff inclusion (diameters of 5.0 and $6.4 \mathrm{~mm}$ for displacement vector components and for shear moduli and strain tensor components, respectively). The results obtained for the 2D AM and the ID AM with demodulation using nonsteered beams are also shown, ie, using the receptions with nonsteered dynamic spherical focusing beams together with the transmissions with nonsteered dynamic spherical focusing beams (see images in figure IE and $F$ in Sumi and Shimizu ${ }^{19}$ ), and the transmission with a nonsteered plane wave (images not shown).

Abbreviations: deg, degrees; freq, frequency; Re, reception; $\mathrm{Tr}$, transmission. 
B Tr, steered plane wave; Re, steered beams

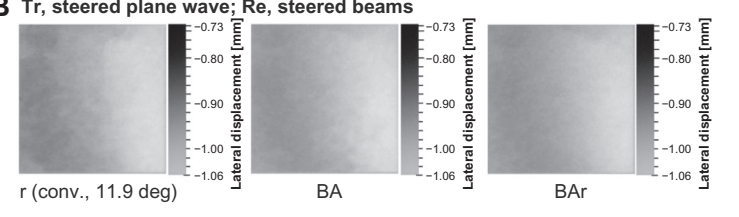

$\mathrm{Tr}$, Non-steered plane wave; Re, steered beams

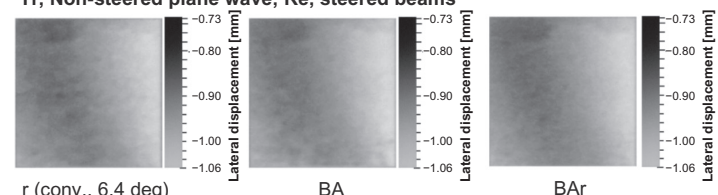

r (conv., 6.4 deg)

BA

BAr

$\mathrm{Tr}$, steered plane wave; Re, steered beams

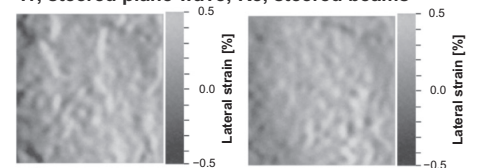

$r$ (conv., $11.9 \mathrm{deg}$ )

BA
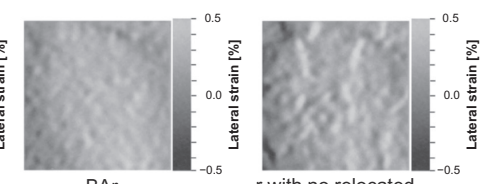

Th, Non-steered plane wave; Re, steered beams
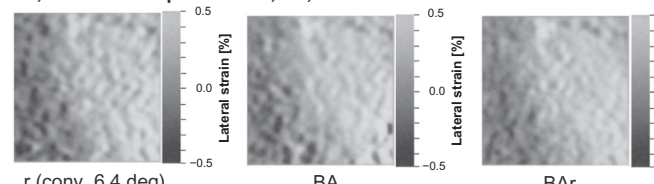

$r$ with no relocated

$r$ (conv, $6.4 \mathrm{deg}$ )

BA

BAr

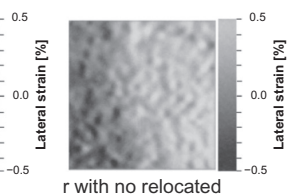

C Tr, steered plane waves; Re, steered beams 2D AM with LM
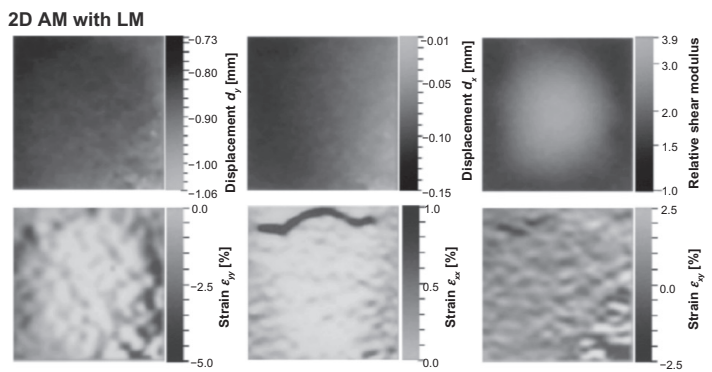

1D AMBA with MCB

1D AM with demodulation and LM
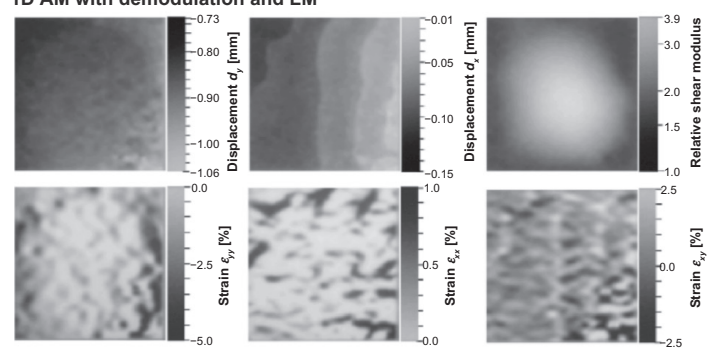

1D AMr $\left(11.9^{\circ}\right)$ with MCB
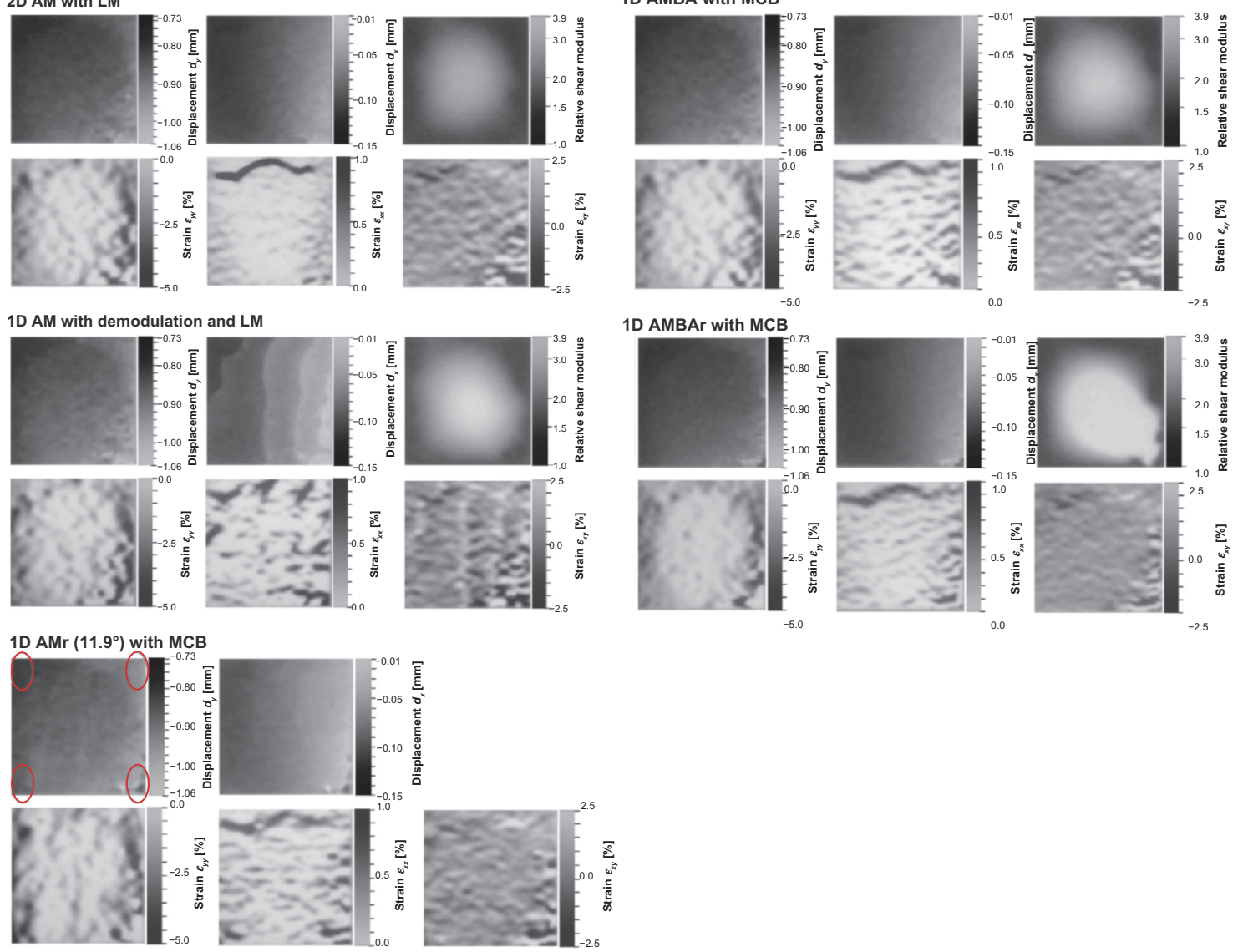

1D AMBAr with MCB

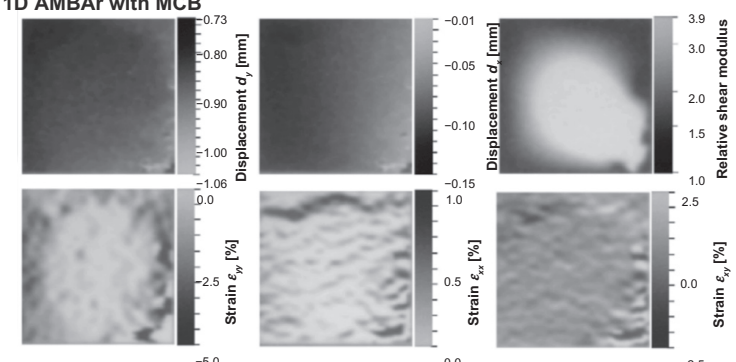

$-5.0$

[ 
$\mathrm{Tr}$, nonsteered plane wave; Re, steered beams

2D AM with LM
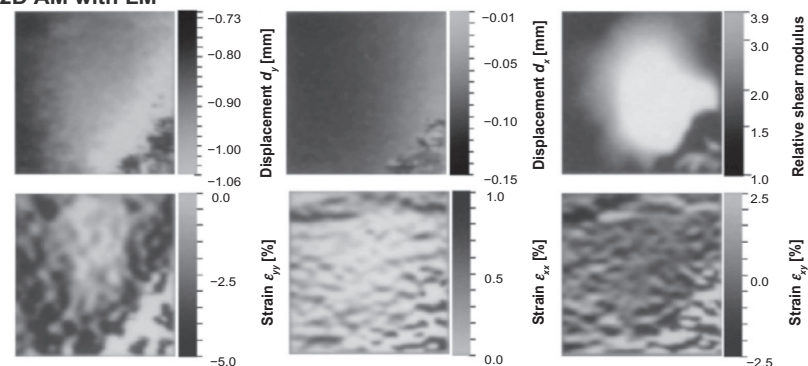

1D AM with demodulation and LM
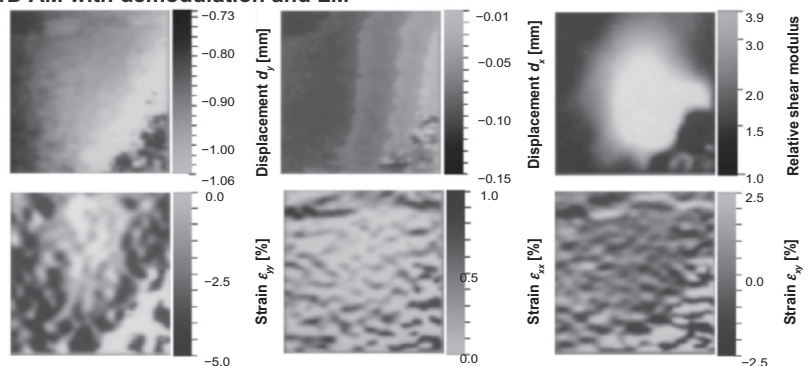

1D $\mathrm{AMr}\left(6.4^{\circ}\right)$ with $\mathrm{MCB}$

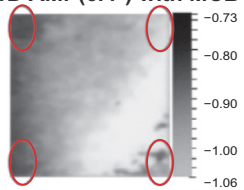

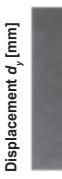
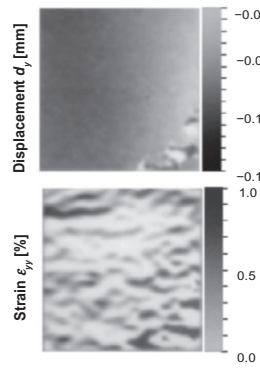
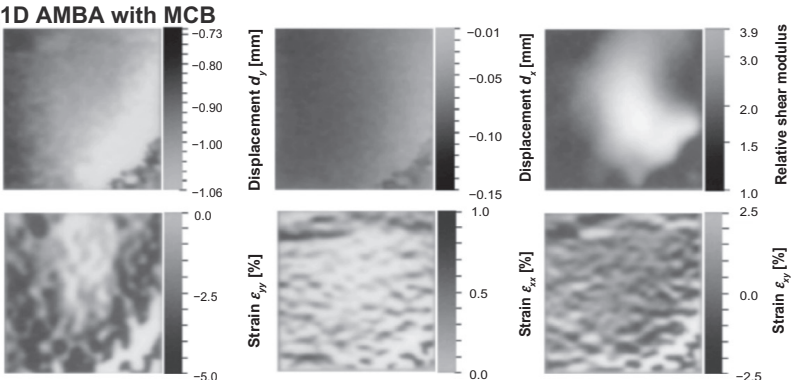

1D AMBAr with MCB
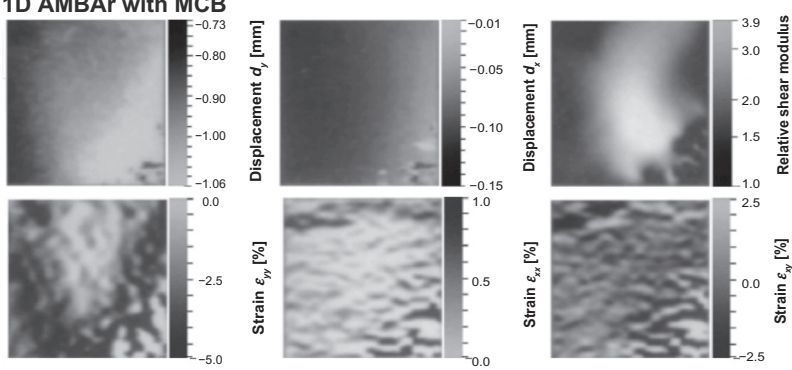
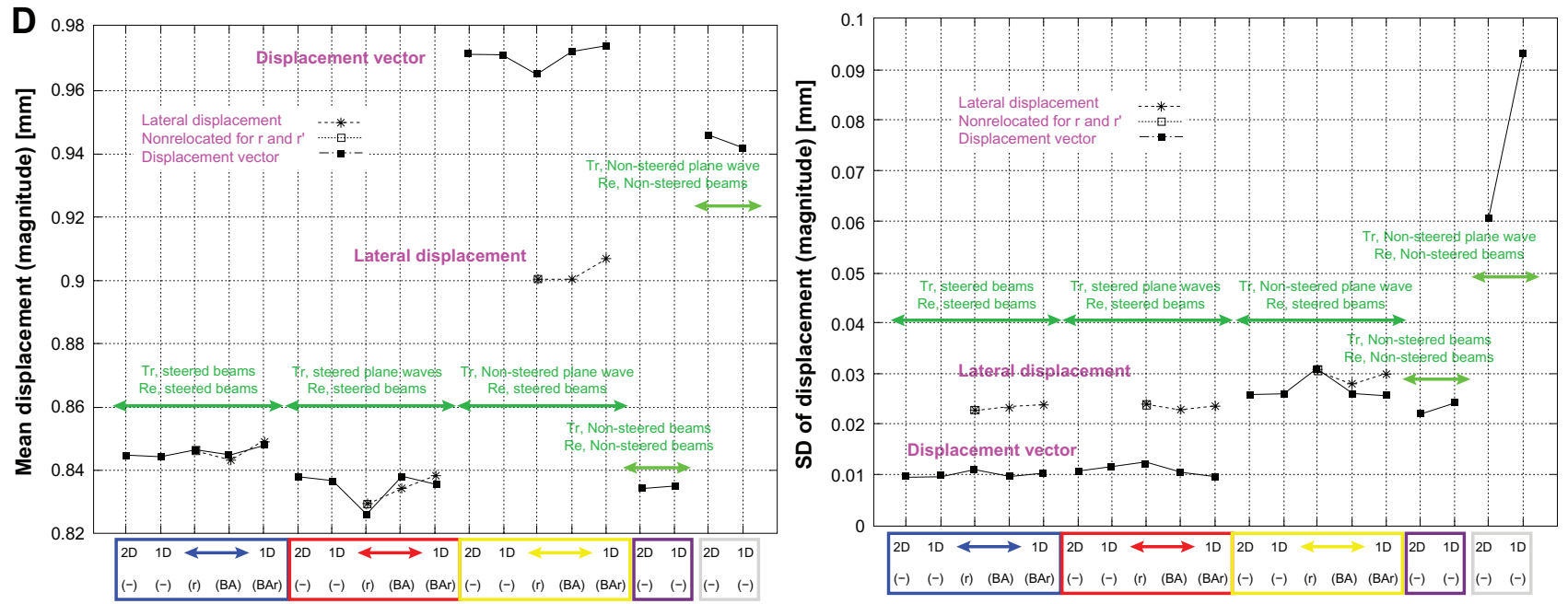

Figure A2 (Continued) 

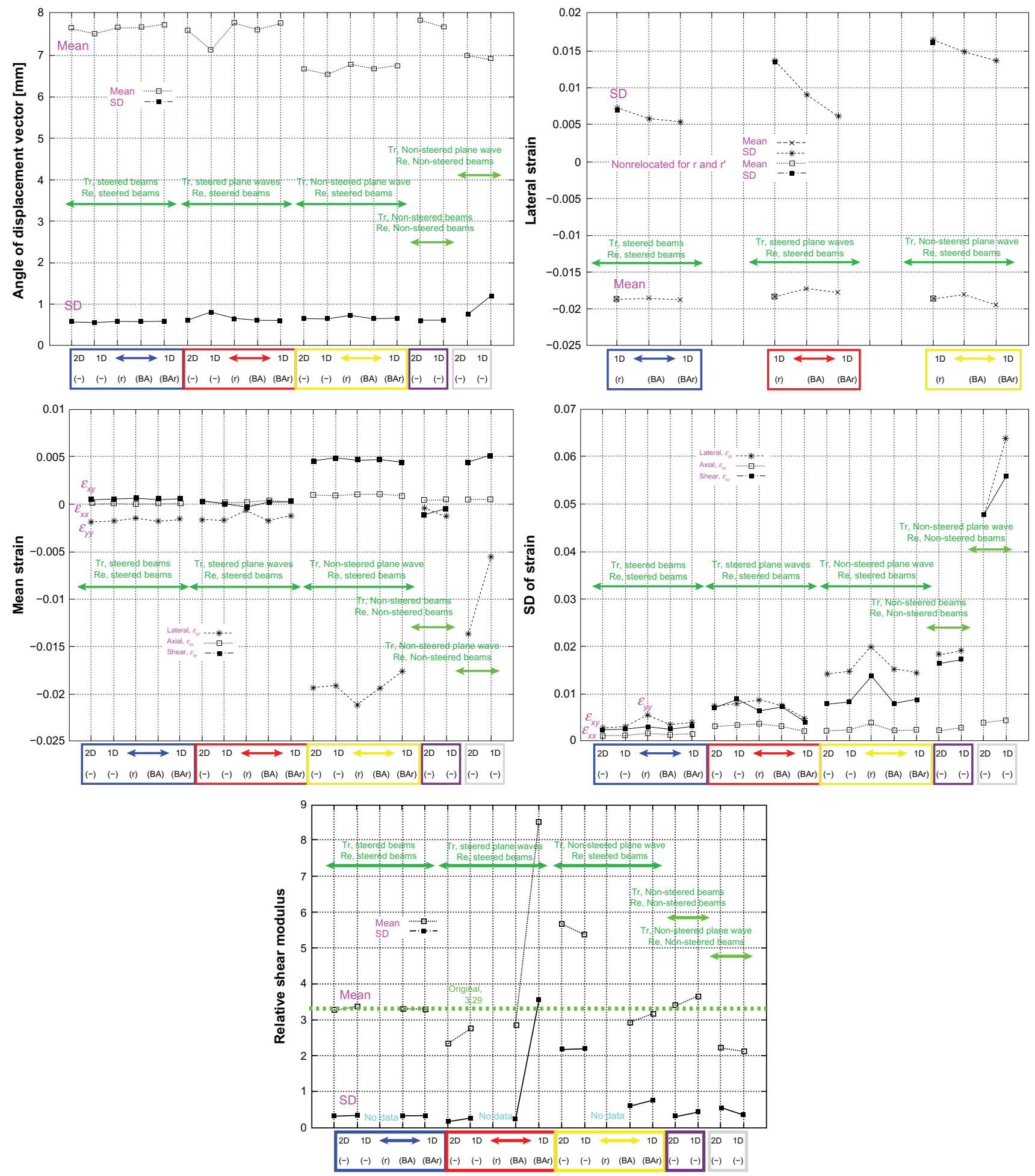

\section{Figure A2}

Reports in Medical Imaging

Dovepress

\section{Publish your work in this journal}

Reports in Medical Imaging is an international, peer-reviewed, open access journal publishing original research, reports, reviews and commentaries on all areas of medical imaging. The manuscript management system is completely online and includes a very quick and fair peer-review system, which is all easy to use. Visit http://www.dovepress.com/testimonials.php to read real quotes from published authors. 